\title{
DRAFT: Numerical investigation of buoyancy-induced flow in a sealed rapidly rotating disc cavity
}

\author{
Feng $\mathrm{Gao}^{1}$, Diogo B. Pitz ${ }^{2,3}$, and John W. Chew ${ }^{1}$ \\ ${ }^{1}$ Thermo-fluid systems UTC, Faculty of Engineering and Physical Sciences, University of Surrey, \\ Guildford, UK \\ ${ }^{2}$ Department of Mechanical Engineering, Federal University of Paraná, Curitiba, Brazil \\ ${ }^{3}$ Faculty of Mechanical Engineering, University of Campinas, Campinas, Brazil
}

October 7, 2019

\begin{abstract}
This paper presents buoyancy-induced flow for a sealed rotating cavity with rotational Rayleigh number $R a$ in the range $10^{7}$ to $10^{9}$. DNS for an incompressible model with the Boussinesq approximation is compared with LES for a compressible gas flow model. The compressible solver's solutions show the shroud Nusselt number scales with $R a^{0.286}$, in close agreement with the corrected experimental correlation and the $R a^{2 / 7}$ scaling for gravitational heat convection between horizontal plates, but differs from the $N u \propto R a^{1 / 3}$ scaling given by the incompressible solver. The shroud thermal boundary layer thickness, based on the root mean square of the temperature fluctuation, can be estimated with $\lambda^{*}=0.5 N u^{-1}$. Velocities scale approximately with $\Omega a \sqrt{\beta \Delta T}$. Disc laminar Ekman layer behaviour is confirmed up to $R a=10^{9}$. An Ekman layer scrubbing effect, associated with the viscous energy dissipation, is considered to be mainly responsible for the difference in $N u$ between the two solvers at $R a=10^{9}$, in spite of rather small Eckert number. The analysis of the turbulent kinetic energy budget shows a dominant constant buoyancy production in the core. The use of the incompressible formulation for the considered problem is restricted by the applicable range of the Boussinesq approximation characterised by the buoyancy parameter $\beta \Delta T$ and neglect of viscous heating and compressibility effects characterised by the Eckert number $E c=\Omega^{2} r_{m}^{2} /\left(C_{p} \Delta T\right)$.
\end{abstract}




\section{Introduction}

The trend in modern jet engine design is towards higher by-pass ratios and higher overall pressure ratios, resulting in smaller engine cores and in turn proportionally larger rotor blade tip clearance. Maintaining a tight blade tip clearance is essential for efficient axial compressor operation, and this is dependent on the radial thermal growth of the rotating discs, which is dominated by buoyancy-induced flow in the centrifugal force field [1]. Most engine disc cavities have an imposed central axial throughflow, but sealed cavities are of direct interest in some cases and provide a canonical test case. The idealised model of buoyancy-induced flow in rotating compressor cavities features two rotating discs, and is radially bounded with a heated shroud and cooled shaft [2]. The heat transfer between the shroud and shaft is by natural convection [3]. If an axial throughflow is introduced between the disc central bore and the shaft, a toroidal vortex is formed at the lower radius region of the cavity, in which heat transfer is by forced convection, but the higher radius region of the cavity remains to be dominated by natural convection [4]. In some configurations, a radial inflow through the shroud may help reduce the thermal response time [5]. Sufficient radial inflow may suppress the buoyancy effects, at the cost of increased use of cooling air [6]. To optimise design and reduce reliance on engine testing, understanding of the related predictive methods for buoyancy effects is required. Previous work on the sealed cavities has left several open questions. The flow is known to be self-organising with a number of cyclonic and anti-cyclonic vortex pairs, but the Nusselt number scaling with rotational Rayleigh number and details of the flow physics are not fully understood.

As buoyancy-induced flow is highly unstable, unsteady, and usually occurs in fairly closed cavities, the laboratory reproduction and measurements of such flows are challenging. The highly unsteady nature and long time scale of the flow also implies difficulties in the numerical modelling of such flows. Recent research [2] reports that the incompressible Navier-Stokes formulation with the Boussinesq approximation, demanding significantly less computing resources than fully compressible formulations, appears to be capable of predicting buoyancy-induced flows in rotating compressor cavities. However, this is not fully confirmed by comparison with available experimental data, as the uncertainties in the measurements are unclear .

The present study considers a sealed rapidly rotating disc cavity experiment as published by Bohn et al. [7]. This isolates the physics of free convection and simplifies the problem. The solutions from an incompressible code using the Boussinesq approximation and a fully compressible gas solver are compared. The validity of use of an incompressible formulation in predicting buoyancy-induced flows in compressor rotor cavities is considered, and results are analysed in detail.

\section{Previous work}

For a wider review of buoyancy-driven flows in rotating cavities, readers are referred to Ref. [1]. Since the time of the review, some further relevant studies have emerged. These include experimental study of buoyancy-induced flow in compressor rotating cavities with axial throughflow $[8,9]$, numerical study of buoyant flows in a sealed rotating annulus $[2,10,11]$ and theoretical modelling of the buoyancy-driven heat transfer inside compressor rotors [12]. Whilst an exhaustive discussion of previous work is not intended here, it is useful to briefly introduce studies relevant to the present work. 
As far as the authors are aware, the only experiment of buoyancy-induced flow in a sealed rapidly rotating annular cavity was published by Bohn et al. [7, 13]. In their work, three airfilled closed annuli were investigated with adiabatic discs and heating of the outer cylindrical walls. The same axial distance between discs and inner cylindrical surface radius was considered in all the configurations. Configuration $\mathrm{A}$ has greater radius at the outer cylindrical surface than Configuration B. Configuration $\mathrm{C}$ has the same geometry as configuration B, but radial plates are installed circumferentially to form 8 identical partitions. Configuration $\mathrm{B}$ has been the subject of recent research and will be further considered here. A schematic diagram of configuration B is given in Fig. 1.

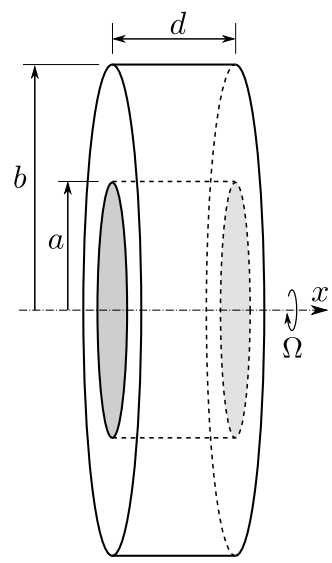

Figure 1: Schematic diagram of Aachen University's configuration B [7].

In considering the test conditions, Bohn et al. [7] noted that the rotational Reynolds number $R e_{\phi}$ and the rotational Rayleigh number $R a$ were not varied independently. Rather, they were related as in Eq. (1). Heat flux was exclusively measured at the outer cylindrical surface and normalised as a Nusselt number $N u$, which is defined as the ratio of the measured convective heat flux to that of the natural heat conduction (given in Eq. (2), where $\kappa$ is the thermal conductivity). $N u$ was measured for a range of $R a$ between $10^{7}$ and $10^{12}$, and a correlation between $R a$ and $N u$ was obtained, as given in Eq. (3).

$$
\begin{gathered}
R e_{\phi}=1.441 R a^{0.557} \\
N u=\frac{\dot{Q}}{\dot{Q}_{\text {cond }}}=\frac{\dot{Q} \ln (b / a)}{2 \pi d \kappa \Delta T} \\
N u=0.317 R a^{0.211}
\end{gathered}
$$

Various studies have compared computational fluid dynamics (CFD) predictions with Bohn et al.'s measurements, with mixed and inconclusive results. In 1998 Bohn and Gier [14] reported some uncertainty as the discs were not perfectly adiabatic, and introduced a correction to the above correlation for $R a \geq 10^{9}$, as given in Eq. (4).

$$
N u_{\text {corr }}=0.0677 R a^{0.297}
$$

Bohn et al. [7] also performed numerical studies on their experimental rig using laminar 
flow equations. Their steady-state simulation showed consistent results with the measurement, in terms of the convective heat transfer described by Nusselt number in Eq. (3). Inspired by Gan et al. [15], Bohn and Gier [14] implemented a low-Reynolds-number $k-\varepsilon$ turbulence model in their code, and predicted stronger heat transfer than the case without turbulence models. It is, however, hard to evaluate if the enhanced heat transfer is physical or due to the spurious effect of the turbulence modelling, or if the trends identified are a consequence of limited mesh resolution.

Sun et al. [3] simulated the same configuration with a time-accurate compressible solver. They reported good agreement, in terms of predicted heat transfer, with the original correlation provided by Bohn et al. [7] in Eq. (3). They also showed the existence of instantaneous large scale flow structures.

Later, in 2005 King et al. [16] carried out 2D simulations on the same configuration using an incompressible Navier-Stokes equation solver with the Boussinesq approximation. Their predicted $N u$ differs from Bohn et al.'s correlation in a rotating annulus, but is in good agreement with the $N u \propto R a^{1 / 3}$ correlations in stationary enclosures under gravity, e.g. by Hollands et al. [17] and Niemela et al. [18]. With regards to the RB convection between horizontal plates under gravity the $N u \sim R a$ scaling exponent is not universal and $N u$ is dependent on $R a$, $\operatorname{Pr}$ and whether the viscous or thermal effect dominates the boundary layer or the bulk of the flow [19]. For the range of $R a$ and $\operatorname{Pr}$ in the present study, a scaling exponent $2 / 7$ is given by Grossmann \& Lohse [19].

Comparing King et al.'s solutions with Sun et al.'s results, Owen and Long [1] attributed the difference to the attenuating effect on heat transfer from the disc boundary layer. Pitz et al. [2], who simulated the 3D geometry with an incompressible DNS (direct numerical simulation) solver using the Boussinesq approximation, also obtained results following $N u \propto R a^{1 / 3}$.

Pitz et al. [11], who used the same code as in [2] but using the large-eddy simulation (LES) mode reported, for the first time, the shroud and disc boundary layer profiles. The disc kinetic boundary layer, based on the position of the maximum root mean square of the radial velocity, showed a scaling proportional to $\Omega^{-0.517}$, similar to that expected for a laminar Ekman layer, $\delta \propto \Omega^{-0.5}$. The instantaneous velocity profiles also behaved as laminar Ekman layers. The thickness of the shroud thermal boundary layer, based on the location of the maximum temperature fluctuations, was found to scale with $R a^{-0.323}$, similar to that for Rayleigh-Bénard (RB) convection.

Saini et al. [20] conducted DNS of Bohn et al.'s rig B for $R a$ between $10^{6}$ and $10^{8}$, using a compressible solver. Some difference with Pitz et al.'s results is reported: a small variation in core temperature was found and the normalised core temperature was reduced as $R a$ increased, in contrast to Pitz et al's results.

Pitz et al. [10] also investigated the onset of convection in the same rotating cavity, using linear stability analysis of the incompressible formulation. They observed convection initiating as counter-rotating vortices evenly distributed in the circumferential direction, similar to RB convection. Pitz et al. also showed the flow was nearly homogeneous in the axial direction except for small variations within the disc boundary layer, as is consistent with the TaylorProudman theorem. The flow was found to drift relative to the rotating frame of reference in the presence of the disc. No drift was observed when the no-slip disc walls were replaced by periodic boundary conditions.

From the review of previous work on the configuration B of Bohn et al.'s rig, we note contrasting conclusions from different researchers, in terms of the shroud heat transfer rate 
(Nusselt number) and the temperature profile. Also, the Boussinesq approximation, which is accepted in geophysics, has not been verified for centrifugal buoyancy-induced gas flows, in which gravitational acceleration is replaced by centripetal acceleration and a Coriolis term emerges. Additionally, viscous heating effects, which are neglected in the Boussinesq approximation framework, may be of importance due to the high speeds involved in the rotating disc cavity configuration.

\section{Test cases considered}

As mentioned above the experimental rig of Bohn et al. [7], configuration B, is considered in this study. An illustration of the disc cavity is given in Fig. 1. The geometry can be described by three characteristic lengths: the radii of the shaft and shroud $(a=0.125 \mathrm{~m}$ and $b=0.24 \mathrm{~m})$, i.e. the inner and outer cylinder surfaces, and the axial distance between the two discs $(d=0.12 \mathrm{~m})$. In the experiment the shroud was heated, whereas the shaft was water-cooled, producing a temperature difference in the radial direction. The two discs were designed to be adiabatic, but the authors later corrected their correlation for non-adiabatic effects. Nusselt numbers were measured for Rayleigh numbers ranging between $10^{7}$ and $10^{12}$. A matrix of the test cases considered in the present study and related parameters is given in Tab. 1, following the correlation in Eq. (1). As $R a$ increases, the buoyancy parameter $\beta \Delta T$ decreases and $R e_{\phi}$ increases. The table also includes the Eckert number $E c$. Where $E c$ is not negligible gas density variations due to pressure (as well as those due to temperature) and/or frictional energy diffusion become significant. As noted, for example, by Kilfoil and Chew [21], and Tang and Owen [12] compressibility leads to a temperature gradient in an isentropic ${ }^{1}$ rotating core. From the values of $E c$ given it appears that frictional and compressive effects may be small, but the results presented later indicate otherwise.

Table 1: Test matrix and related parameters.

\begin{tabular}{llll}
\hline$R a$ & $\beta \Delta T$ & $R e_{\phi}$ & $E c$ \\
\hline $10^{7}$ & 0.174 & $1.14 \times 10^{4}$ & $3.82 \times 10^{-5}$ \\
$10^{8}$ & 0.134 & $4.12 \times 10^{4}$ & $6.60 \times 10^{-4}$ \\
$10^{9}$ & 0.103 & $1.48 \times 10^{5}$ & $1.13 \times 10^{-2}$ \\
\hline
\end{tabular}

\section{$4 \quad$ Numerical methods}

As one purpose of the present study is to explore the validity of using an incompressible formulation with the Boussinesq approximation for rotational buoyancy-induced flows, both incompressible and compressible solvers are used. The features of both solvers are briefly introduced in this section, and the detailed governing equations are given in Appendix A. The specific numerical settings and boundary conditions of the simulations are also given in this section.

\footnotetext{
${ }^{1}$ The term "adiabatic" is used by Tang and Owen [12]
} 


\subsection{Hydra}

Hydra is an in-house flow solver, considering the fully compressible form of the Navier-Stokes equations in a Cartesian coordinate system, developed by Rolls-Royce plc and its university partners. It uses a finite volume method, on node-vertex unstructured grids. The spatial discretisation is realised using monotone upwind schemes for conservation laws (MUSCL) based on a modified Roe scheme. In the version used here, second-order accuracy is achieved by a linear reconstruction of primitive flow variables as described by Amirante and Hills [22]. Regarding the temporal interpolation, an explicit 3-stage Runge-Kutta scheme is adopted. Air is simulated in this study as a perfect gas, and the heat capacity ratio $\gamma$ is fixed at 1.4. The dynamic viscosity is variable following Sutherland's law. The Prandtl number $\operatorname{Pr}$ and the specific heat capacity at constant pressure $C_{p}$ are fixed as 0.7 and $1004.15 \mathrm{~J} \cdot \mathrm{kg}^{-1} \cdot \mathrm{K}^{-1}$, respectively. The heat conductivity is calculated as $\kappa=\mu C_{p} / \operatorname{Pr}$. The natural heat conduction $\dot{Q}_{\text {cond }}$ in Eq. (2) is estimated with property values at the shaft temperature.

\subsection{Semtex}

Semtex is an open-source code, solving the incompressible Navier-Stoke equations [23] in either Cartesian or cylindrical coordinate systems. This code uses a spectral element-Fourier method, combining the flexibility of geometry of the finite element method and high-order accuracy of spectral methods. High-order Lagrange polynomials are employed, in each parametrically mapped quadrilateral element within a 2D plane, to achieve spectral accuracy. Fourier expansions are used along the third direction, which is required to be homogeneous. A second-order, semi-implicit, stiffly stable scheme introduced by Karniadakis et al. [24] is implemented for time discretisation. Semtex parallelises only along the homogeneous direction, with a minimum constraint of 2 Fourier planes per CPU core.

Unlike compressible solvers, where the density variations and energy equation are directly included, the Boussinesq approximation and the temperature transport equation are implemented to model buoyancy-induced flows. Equations are detailed in Appendix A.2.

\subsection{Boussinesq approximation}

The Boussinesq approximation was proposed by and named after Joseph Valentin Boussinesq [25], to consider the buoyancy-driven flow under gravity in geophysics using the incompressible Navier-Stokes equations. The approximation is only applied to the terms multiplied by the gravitational acceleration in the momentum equation. Density is assumed to vary linearly with temperature within limits of the approximation, which is typically assumed valid for $\beta \Delta T \lesssim 0.2$. In the present study this approximation is applied to the centrifugal term. More details can be found in Appendix A.2.

\subsection{Numerical settings and boundary conditions}

The Hydra calculations are performed in LES mode, using the standard Smagorinsky model $\left(C_{s}=0.08\right)$ and the van Driest damping function. For Semtex simulations a DNS approach is used. 
With both solvers, flow is simulated in the rotating frame of reference. The discs are set as no-slip adiabatic walls, whilst the shaft and shroud surfaces are defined as no-slip isothermal walls with fixed temperatures. The shaft was cooled by water in the experiment. Therefore, a fixed shaft temperature $T_{a}=288 \mathrm{~K}$ is applied to the CFD. The thermal expansion ratio $\beta$ is defined as the inverse of the arithmetic mean of the shroud and shaft temperatures, i.e. $\beta=2 /\left(T_{a}+T_{b}\right)$. With these relations the shroud temperature $T_{b}$ can be calculated following $\beta \Delta T$ in Tab. 1. The shaft and shroud temperatures, in Hydra simulations, are $288 \mathrm{~K}$ and $T_{b}$, respectively. In Semtex the reference temperature is defined on the shaft, and the shroud temperature is $\Delta T$ higher. The absolute value of the reference temperature is not important in the incompressible formulation, as it vanishes in the derivatives of the temperature transport equation.

Whilst grid convergence studies have been conducted for all the configurations presented in this paper, only those at $R a=10^{9}$ are presented here. The grids used and some key results are given in Tabs. 2 and 3 for Hydra and Semtex, respectively. The near-wall grid spacing $\Delta_{w}$ is the same on all walls, and represents the minimum grid spacing in all cases. The tables also give the maximum mesh spacing and numbers of grid points or elements, as appropriate. The convergence of grid resolution is evaluated through the shroud Nusselt number $N u$ and the mean core temperature $T_{\text {core }}^{*}$ As the Hydra mesh is refined convergent behaviour is observed. Although some grid resolution dependency is still seen on the fine mesh, the fact that the changes of $N u$ and $T_{\text {core }}^{*}$ from medium to fine meshes are much smaller suggests reasonably converged results are being obtained at affordable computing costs. The grid resolution dependency is significantly smaller than the difference in shroud $N u$ between the two codes. The ratio of the subgrid scale (SGS) dissipation and numerical dissipation to the total dissipation is plotted against the radius in Fig. 2 at the mid-axial position for the fine grid solution. This plot indicates that the SGS and numerical dissipation are active and simulate an appropriate amount of the total dissipation, as is required for LES [26].

With Semtex, a DNS study is performed with much finer grids. The number of elements is fixed as 484 . The mesh improvement is achieved by increasing the polynomial order in the meridional plane and the number of Fourier planes in the circumferential direction. Some non-monotonic change in $N u$ with grid was first observed for the first three mesh levels at $R a=10^{9}$, and was also observed in Ref. [2]. The additional finer mesh level confirms the nonmonotonic behaviour and convergence with grid resolution. Detailed comparison (not presented for brevity) between the fine and finer mesh results shows no noticeable difference, and the fine mesh results are presented in this paper. The errors of the overall heat balance between the cylindrical surfaces at $R a=10^{9}$ are $1.7 \%$ and $3.8 \%$ for Hydra and Semtex, respectively. The energy generation due to the mean angular momentum error is less than $0.5 \%$ of the error of the overall heat balance. The final mesh resolutions are given in Tab. 4 for both Hydra and Semtex simulations.

\section{$5 \quad$ Results and discussions}

Instantaneous radial and tangential velocity, and temperature contours obtained with Hydra are plotted in Fig. 3. The temperature contour from Semtex is also shown in the figure. The contours show normalised values of the properties illustrated. Alternating radially inward and outward flow is observed in the radial velocity contour in subplot (a). Along with the tangential 
Table 2: Convergence of grid resolution for $R a=10^{9}$, with Hydra. $\Delta_{w}$ : near-wall grid spacing. $N_{x, r, \theta}$ : number of grid points in the axial, radial and tangential directions. $N_{\text {tot }}$ : total number of grid points. $\Delta()_{\max }$ : maximum grid spacing. $N u$ : shroud Nusselt number. $T_{\text {core }}^{*}$ : mean core temperature. M: million.

\begin{tabular}{lllllllllll}
\hline \multirow{2}{*}{ Grid } & $\begin{array}{l}\Delta_{w} \\
{[\mathbf{m m}]}\end{array}$ & $N_{x}$ & $N_{r}$ & $N_{\theta}$ & $N_{\text {tot }}$ & $\begin{array}{l}\Delta x_{\max } \\
{[\mathbf{m m}]}\end{array}$ & $\begin{array}{l}\Delta r_{\max } \\
{[\mathbf{m m}]}\end{array}$ & $\begin{array}{l}\Delta(r \theta)_{\max } \\
{[\mathbf{m m}]}\end{array}$ & \multirow{2}{*}{$N u$} & $T_{\text {core }}^{*}$ \\
\hline Coarse & 0.4 & 51 & 51 & 200 & $\sim 0.5 \mathrm{M}$ & 4.19 & 3.91 & 7.54 & 34.7 & 0.640 \\
Medium & 0.1 & 71 & 71 & 400 & $\sim 2.0 \mathrm{M}$ & 3.94 & 3.62 & 3.77 & 39.0 & 0.652 \\
Fine & 0.05 & 85 & 85 & 600 & $\sim 4.3 \mathrm{M}$ & 3.16 & 2.97 & 2.51 & 40.3 & 0.654 \\
\hline
\end{tabular}

Table 3: Convergence of grid resolution for $R a=10^{9}$, with Semtex. $\Delta_{w}$ : near-wall grid spacing. $N_{\mathrm{el}}$ : number of elements in a meridional plane. $P$ : polynomial order. $N_{\theta}$ : number of grid points in the tangential direction. $N_{\text {tot }}$ : total number of grid points. $\Delta()_{\text {max }}$ : maximum grid spacing. $N u$ : shroud Nusselt number. $T_{\text {core }}^{*}$ : mean core temperature. M: million.

\begin{tabular}{lllllllllll}
\hline Grid & $\begin{array}{l}\Delta_{w} \\
{[\mathbf{m m}]}\end{array}$ & $N_{\mathrm{el}}$ & $P$ & $N_{\theta}$ & $N_{\text {tot }}$ & $\begin{array}{l}\Delta x_{\max } \\
{[\mathbf{m m}]}\end{array}$ & $\begin{array}{l}\Delta r_{\max } \\
{[\mathbf{m m}]}\end{array}$ & $\begin{array}{l}\Delta(r \theta)_{\max } \\
{[\mathbf{m m}]}\end{array}$ & \multirow{2}{*}{$N u$} & $T_{\text {core }}^{*}$ \\
\hline Coarse & 0.14 & 484 & 5 & 128 & $\sim 1.5 \mathrm{M}$ & 2.98 & 2.60 & 11.8 & 56.5 & 0.646 \\
Medium & 0.06 & 484 & 8 & 256 & $\sim 7.9 \mathrm{M}$ & 1.90 & 1.65 & 5.89 & 49.9 & 0.645 \\
Fine & 0.04 & 484 & 10 & 512 & $\sim 24.8 \mathrm{M}$ & 1.55 & 1.35 & 2.95 & 51.5 & 0.650 \\
Finer & 0.02 & 484 & 13 & 640 & $\sim 52.3 \mathrm{M}$ & 1.22 & 1.06 & 2.36 & 51.0 & 0.643 \\
\hline
\end{tabular}

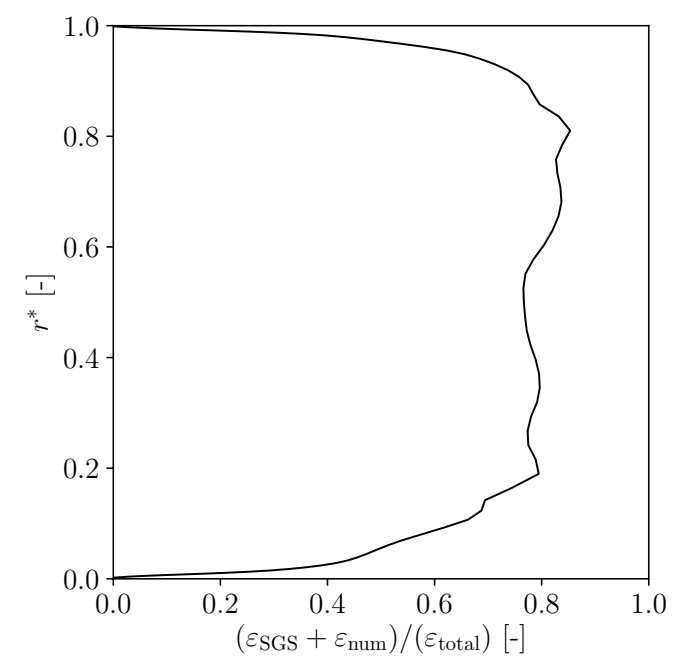

Figure 2: Ratio of the SGS dissipation and numerical dissipation to the total dissipation at mid-axial position obtained with Hydra for $R a=10^{9}$. Results are averaged in time and in the circumferential direction. A simple smoothing function is applied to remove some grid-to-grid oscillation. $r^{*}=(r-a) /(b-a)$. 
Table 4: Fine mesh resolutions. $\Delta_{w}$ : near-wall grid spacing. $N_{x, r, \theta}$, number of nodes in the axial, radial and azimuthal direction; $N_{\text {tot }}$, total number of grid points; $N_{\text {el }}$, number of elements in a meridional plane; $P$, polynomial order; $\mathrm{M}$, million.

\begin{tabular}{|c|c|c|c|c|c|c|c|c|c|c|}
\hline \multirow[b]{2}{*}{$R a$} & \multicolumn{5}{|c|}{ Hydra (LES) } & \multicolumn{5}{|c|}{ Semtex (DNS) } \\
\hline & $\Delta_{w}[\mathrm{~mm}]$ & $N_{x}$ & $N_{r}$ & $N_{\theta}$ & $N_{\text {tot }}$ & $\Delta_{w}[\mathrm{~mm}]$ & $N_{\mathrm{el}}$ & $P$ & $N_{\theta}$ & $N_{\text {tot }}$ \\
\hline $10^{7}$ & 0.2 & 61 & 61 & 200 & $\sim 0.7 \mathrm{M}$ & 0.077 & 484 & 7 & 384 & $\sim 9.1 \mathrm{M}$ \\
\hline $10^{8}$ & 0.1 & 75 & 75 & 600 & $\sim 3.4 \mathrm{M}$ & 0.077 & 484 & 7 & 384 & $\sim 9.1 \mathrm{M}$ \\
\hline $10^{9}$ & 0.05 & 85 & 85 & 600 & $\sim 4.3 \mathrm{M}$ & 0.04 & 484 & 10 & 512 & $\sim 24.8 \mathrm{M}$ \\
\hline
\end{tabular}

flow patterns in subplot (b) these indicate four cyclonic and anti-cyclonic vortex pairs in the annulus. The radial velocities indicate radial jets at the same positions as the hot and cold arms in subplot (c). Similar temperature contours are given by Hydra and Semtex in subplots (c) and (d), and these are in broad agreement with previous studies, both for compressible $[3,20]$ and incompressible $[11,16]$ models. Though not shown in the figure, velocity contours obtained with Semtex are in good agreement with those shown in Figs. 3 (a) and (b).

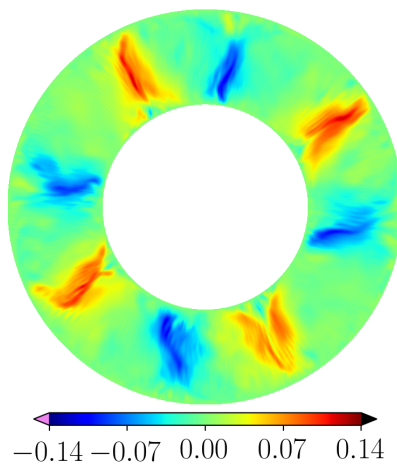

(a) $v_{r}^{*}$, Hydra

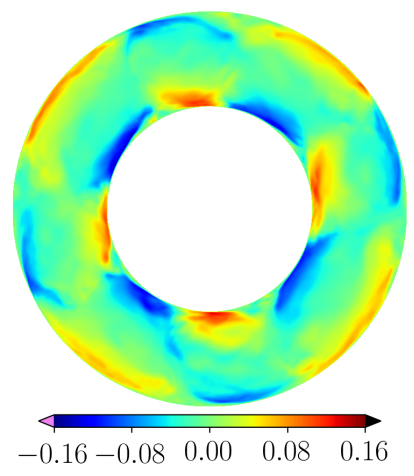

(b) $v_{\theta}^{*}$, Hydra

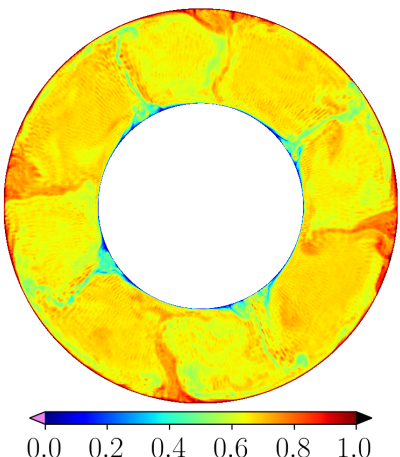

(c) $T^{*}$, Hydra

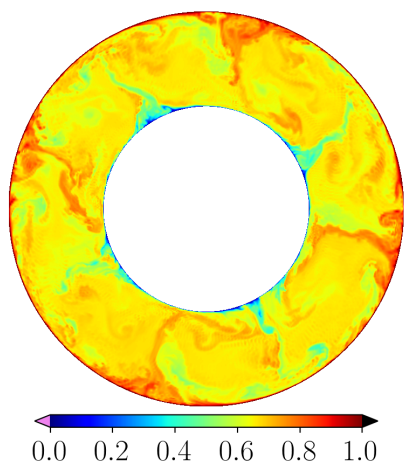

(d) $T^{*}$, Semtex

Figure 3: Instantaneous flow field at $R a=10^{9}$. Plots are extracted at the mid-axial position between the discs. Rotation is anti-clockwise. $v_{r}^{*}=v_{r} /(\Omega r) . v_{\theta}^{*}=v_{\theta} /(\Omega r) . T^{*}=\left(T-T_{a}\right) /\left(T_{b}-\right.$ $\left.T_{a}\right)$.

\subsection{Nusselt number}

Figure 4 shows the predicted shroud Nusselt numbers from Hydra and Semtex, which are also listed in Tab. 5. The percentage differences in $N u$ between the two codes are given on the graph. Also plotted on the graph are Bohn and co-workers' original [7] and corrected [14] (for $R a \geq 10^{9}$ ) correlations for the rig considered in the present study. Although the correction was not intended for use at the lower Rayleigh number, it is included to give an indication of the possible experimental uncertainty. The two correlations of Bohn et al.'s rig cross near $R a=10^{8}$. The Hydra and Semtex solutions show closer agreement with the original correlation at $R a=10^{7}$ but match better with the corrected correlation at $R a=10^{9}$, consistent with the interpretation of the experimental data [7, 14]. In correlating the Hydra solutions a scaling of $N u \propto R a^{0.286}$ is obtained, showing close agreement with the scaling in the corrected correlation 
provided in Eq. (4) by Bohn and Gier [14] and the $R a^{2 / 7}$ scaling for RB convection [19] for the $R a$ and $\operatorname{Pr}$ range investigated in the present study. The $N u$ predicted by Semtex follows a $R a^{0.329}$ scaling, in agreement with Pitz et al. [2] and Hollands et al.'s correlation for natural convection under gravity [17] (giving $N u \propto R a^{1 / 3}$ at high $R a$ values). Good agreement between the two solvers is observed at the low $R a$ value. As $R a$ is increased the difference between the two solvers rises to $21.8 \%$. Note that increasing $R a$ as in the experiment reduces $\beta \Delta T$, which might be expected to be favourable for the Boussinesq approximation. This implies that there are other mechanisms driving the difference, as will be discussed later. Note also that uncertainty in the experiment might contribute to the discrepancy with the experimental correlation.

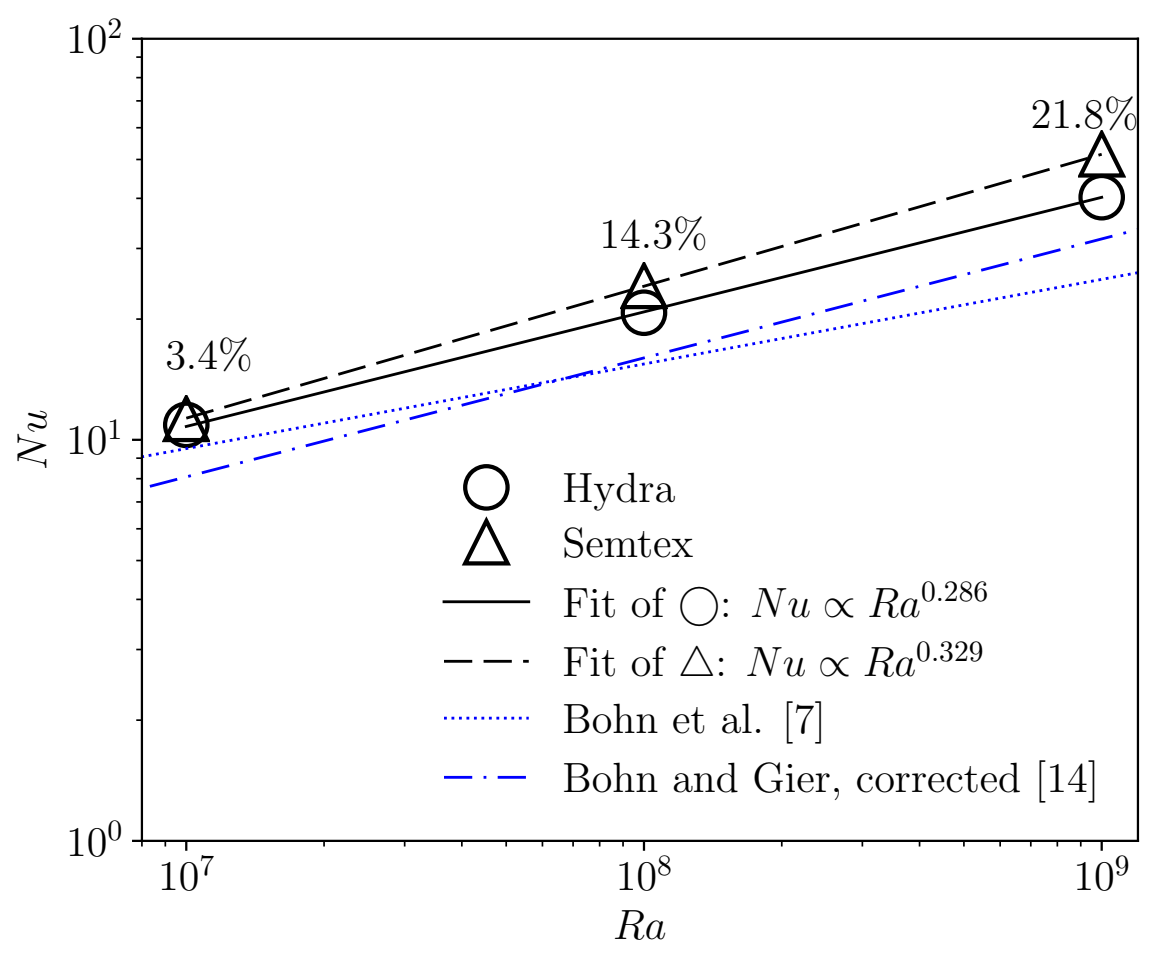

Figure 4: Shroud $N u$ versus $R a$, for Bohn et al.'s rig condition. The percentages shown correspond to differences between the Hydra and Semtex solutions.

Table 5: Shroud Nusselt number $N u$. Comparison between Hydra and Semtex.

\begin{tabular}{llll}
\hline & $R a=10^{7}$ & $R a=10^{8}$ & $R a=10^{9}$ \\
\hline Hydra & 10.89 & 20.74 & 40.26 \\
Semtex & 11.27 & 24.19 & 51.51 \\
\hline
\end{tabular}

\subsection{Shroud and shaft boundary layers}

The thermal and kinetic boundary layer parameters discussed here are mean results, averaged in time and the azimuthal direction. According to the Taylor-Proudman theorem and also confirmed by Pitz et al. [10], negligible axial variation is found away from disc boundary layers. Therefore, the mean profiles are also averaged axially between $x^{*}=x / d=0.25$ and 0.75 . 
Profiles of the mean temperature and the temperature fluctuation root mean square are plotted in Fig. 5 for Semtex solutions and in Fig. 6 for Hydra results. Uniform core temperatures are observed in both Semtex and Hydra solutions, for the values of $R a$ considered. Figure 5 shows Semtex core temperatures slightly decrease with the increase of $R a$, opposite to Pitz et al.'s results [11] using the same solver but with implicit LES and coarser meshes. This indicates some uncertainty regarding the use of the spectral vanishing viscosity. The profile of $T_{\mathrm{rms}}^{*}$ shows decreasing values in the core and thinning thermal boundary layers as $R a$ increases, as is consistent with Pitz et al.'s results [11]. The core temperature is almost invariant in Hydra solutions, for the range of $R a$ simulated. Similar profiles of $T_{\mathrm{rms}}^{*}$ to Semtex are obtained with Hydra. The profiles of the two solvers are compared in Fig. 7 for $R a=10^{9}$, showing good agreement. Note, however, that the difference in shroud $N u$ between the compressible and Boussinesq models reaches its maximum value at this $R a$. Zoomed views at the shroud are also provided as insets in Fig. 7. Slight differences can be observed. The predicted $N u$ depends on the gradient of mean temperature on the shroud, which shows small differences accounting for much of the $21.8 \%$ difference in $N u$. The increased thermal conductivity of the air at the heated shroud in Hydra also contributes to the difference in $N u$ predictions. For $T_{\text {rms }}^{*}$ differences between Hydra and Semtex at the shroud are seen in the zoomed inset. The shroud thermal boundary layer is thicker in Hydra's compressible flow solution.
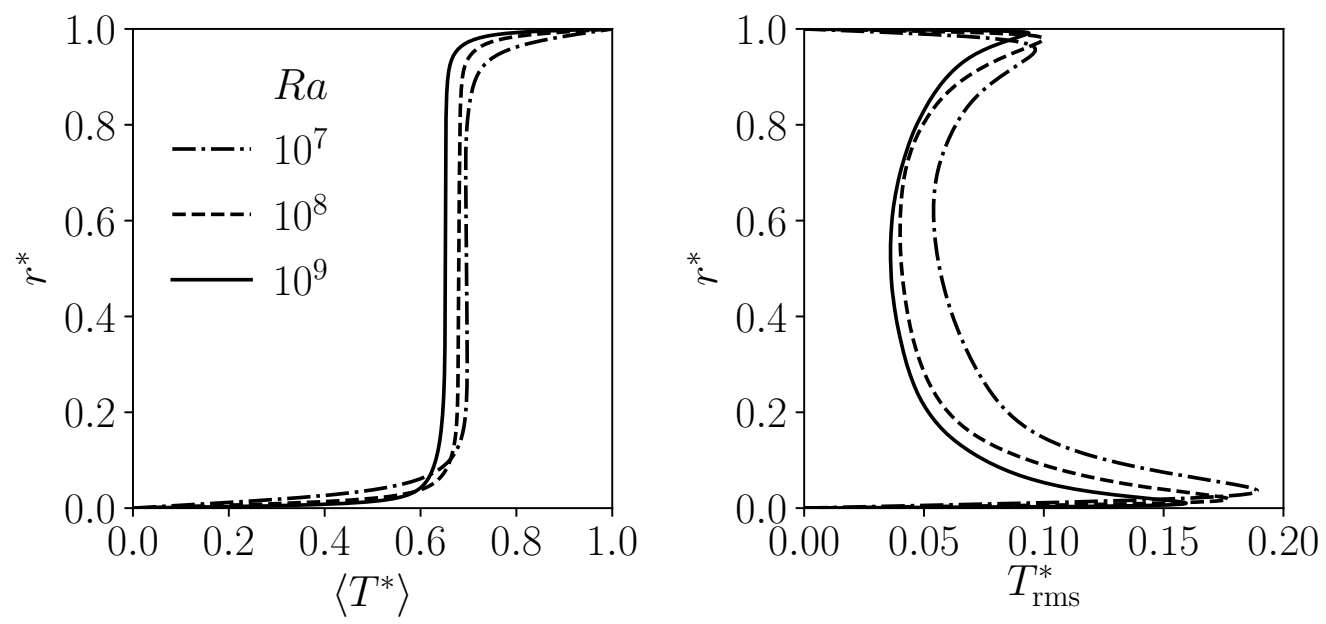

Figure 5: Thermal boundary layers for different $R a$, obtained from Semtex. Left: mean nondimensional temperature profiles. Right: root mean square of the non-dimensional temperature fluctuation. Data are averaged in time, in the azimuthal direction and between $x^{*}=0.25$ and 0.75 .

A shroud thermal boundary layer thickness $\lambda$ is determined by the position of peak $T_{\text {rms }}$. The thermal boundary layer profiles normalised by $T_{\mathrm{rms} \text {,max }}$ and $\lambda$, and obtained with Hydra, are plotted in the left subplot of Fig. 8 for the three $R a$ values considered. A good match between the three profiles is observed. A similar match is also achieved with Semtex, in agreement with Pitz et al.'s observation. The normalised shroud thermal boundary layer thickness $\lambda^{*}$ $(=\lambda /(b-a))$ based on $T_{\text {rms }}^{*}$, from both Hydra and Semtex, is plotted against $R a$, in the right subplot of Fig. 8. Closest agreement between the two solvers is seen at $R a=10^{7}$, and the difference develops progressively as $R a$ is increased. This is consistent with the scaling observed for $N u$. As introduced by Grossmann \& Lohse [19] the non-dimensional thermal boundary layer 

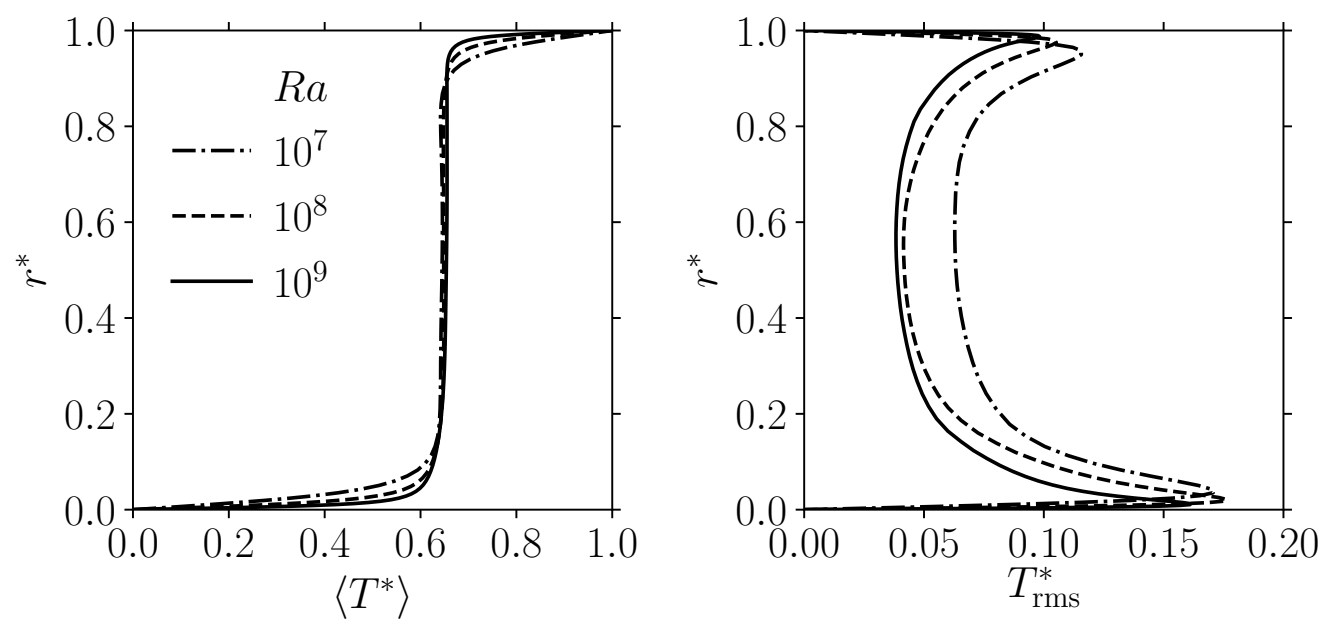

Figure 6: Thermal boundary layers for different $R a$, obtained with Hydra. Left: mean nondimensional temperature profiles. Right: root mean square of the non-dimensional temperature fluctuation. Data are averaged in time, in the azimuthal direction and between $x^{*}=0.25$ and 0.75 .
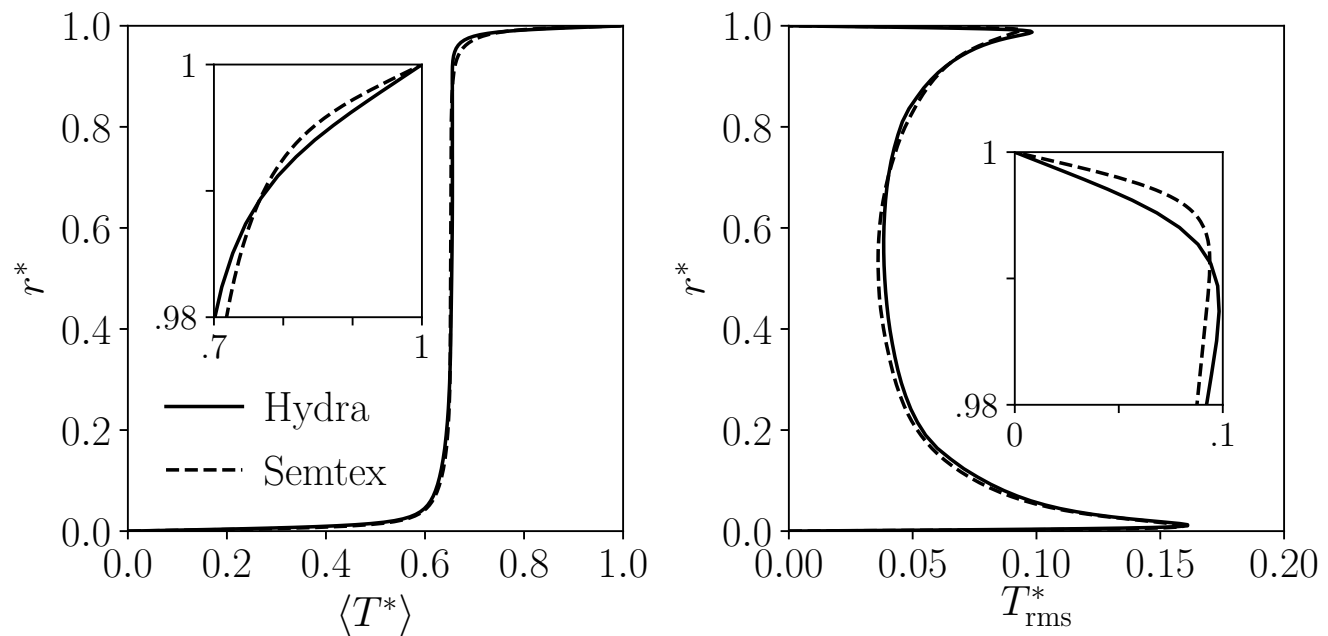

Figure 7: Thermal boundary layer at $R a=10^{9}$, comparison between Hydra and Semtex. Left: mean non-dimensional temperature profiles. Right: root mean square of the non-dimensional temperature fluctuation. Data are averaged in time, in the azimuthal direction and between $x^{*}=0.25$ and 0.75 . Insets show zoomed view at shroud. 
thickness in RB convection can be estimated as $\lambda^{*}=0.5 N u^{-1}$. These are plotted in the right subplot of Fig. 8, and show remarkably good agreement with the corresponding data. Note that the determination of the position of peak $T_{\text {rms }}$ relies on the mesh resolution in Hydra, which introduces relatively larger uncertainty at $R a=10^{7}$ due to thicker thermal boundary layer and mesh expansion away from the wall.
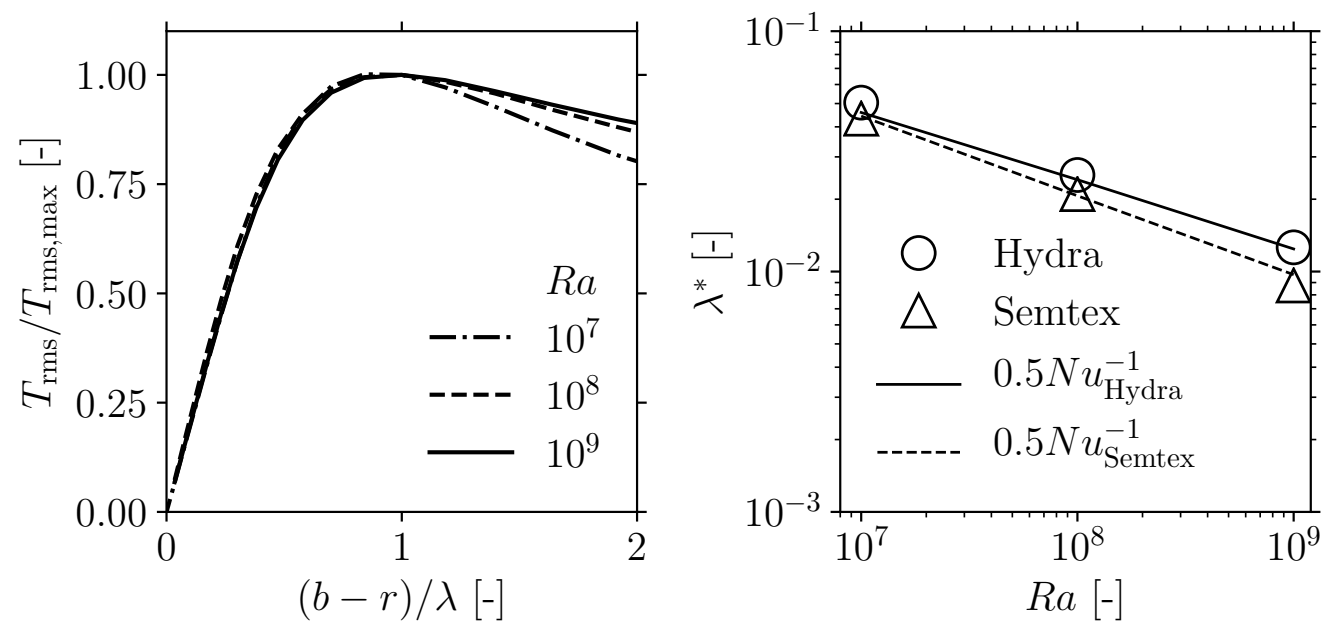

Figure 8: Normalised shroud thermal boundary layer from Hydra's solution (left), and shroud boundary layer thickness $\lambda^{*}$ versus $R a$ (right) based on $T_{\mathrm{rms}}^{*} \cdot \lambda^{*}=\lambda /(b-a)$. Data are averaged in time, in the azimuthal direction and between $x^{*}=0.25$ and 0.75 .

The root mean square of radial and azimuthal velocity fluctuations is presented here to illustrate the shaft and shroud kinetic boundary layer. The profiles of $v_{r, \text { rms }}$ normalised with the tangential speed on the shaft are shown on the left subplot in Fig. 9. Maximum values are seen at the mid-radius, and decrease as $R a$ is increased. Considering the buoyancy force as the driving force and further normalising these profiles with $\sqrt{\beta \Delta T}$, a better match is achieved between results at different values of $R a$. Applying the same normalisation to the azimuthal velocity fluctuation, a similar trend can be observed, as shown in Fig. 10. Note that peaks are located near the shroud and shaft, and the kinetic boundary layer thickness thins with increase of $R a$, as is consistent with the thermal boundary layer. The plots in Figs. 9 and 10 suggest that velocity scales roughly with $\Omega a \sqrt{\beta \Delta T}$. The results from Semtex are not presented here but give the same conclusion. Velocity profiles from Hydra and Semtex, for $R a=10^{9}$, are compared in Fig. 11. Qualitative agreement is observed, but Semtex shows somewhat higher values.

\subsection{Disc kinetic boundary layers}

\subsubsection{Averaged velocity profiles}

Mean radial velocity profiles and the corresponding root mean squares of fluctuations are obtained by averaging in time and the azimuthal direction. These profiles at $R a=10^{9}$ are plotted in Fig. 12. Also shown on the subplots is the thickness for a laminar Ekman layer $(\pi / d)(\Omega / \nu)^{-0.5}$, which scales with the Ekman depth $(\Omega / \nu)^{-0.5}$. 

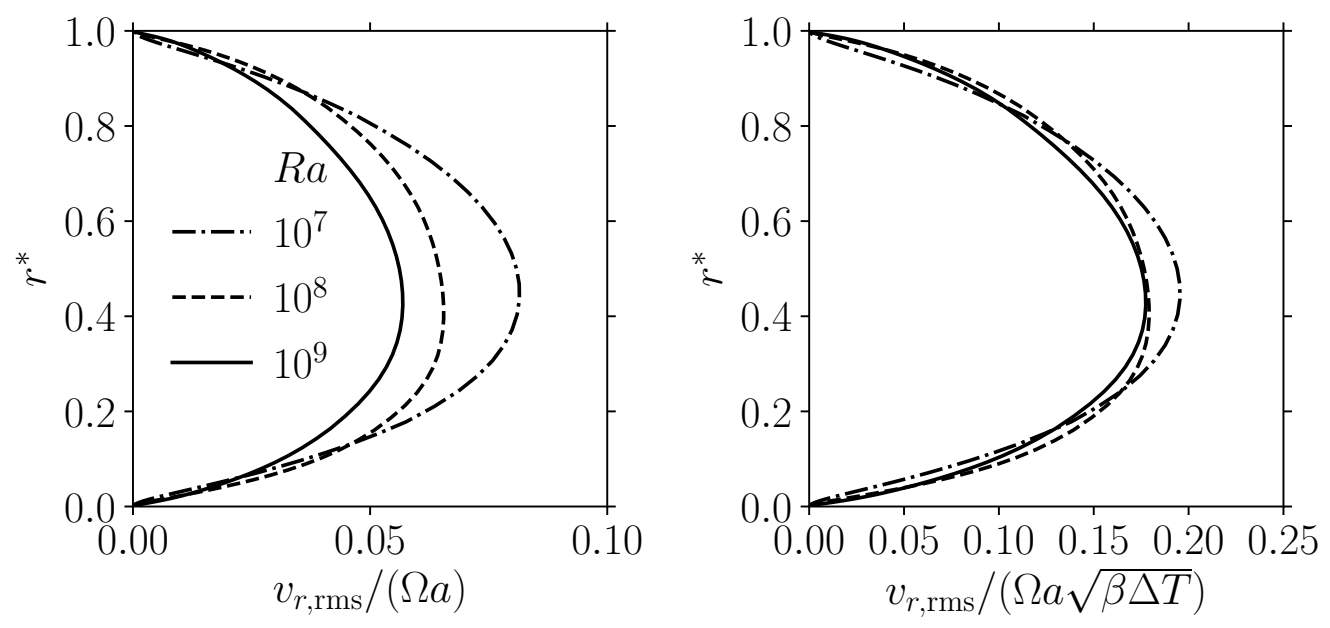

Figure 9: Profiles of radial velocity fluctuations' root mean square, obtained with Hydra. Left: normalised with the tangential speed on the shaft. Right, further normalised with $\sqrt{\beta \Delta T}$. Data are averaged in time, in the azimuthal direction and between $x^{*}=0.25$ and 0.75 .
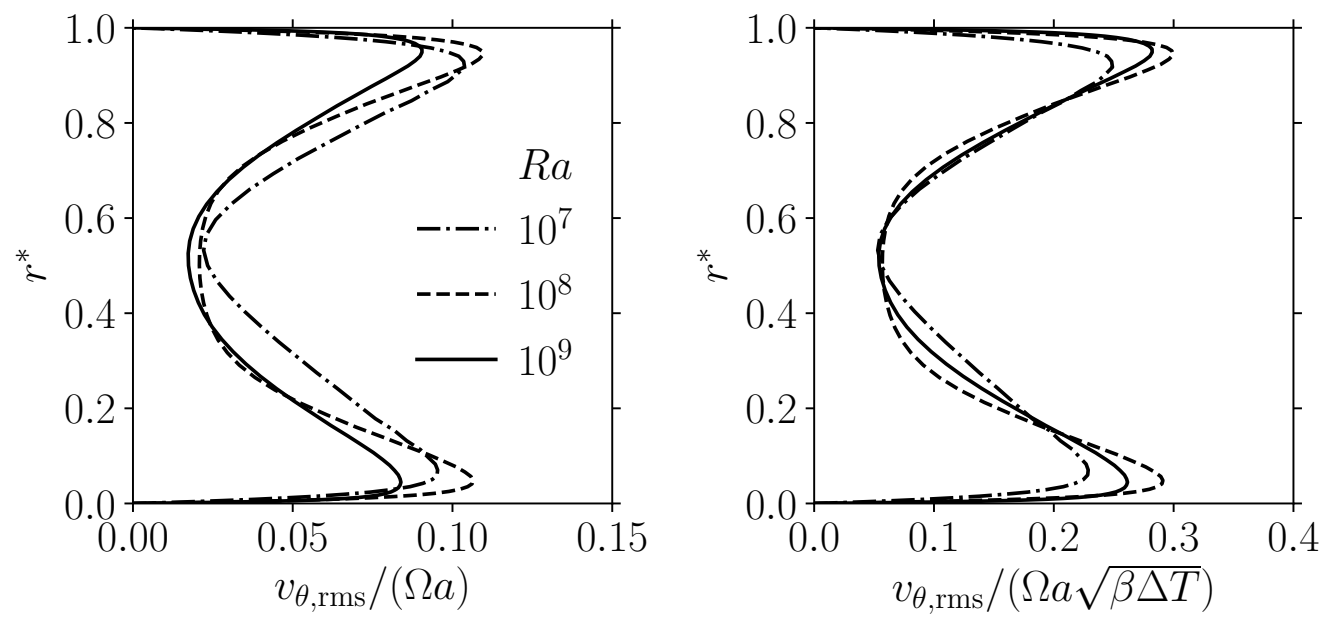

Figure 10: Profiles of azimuthal velocity fluctuations' root mean square, obtained with Hydra. Left: normalised with the tangential speed on the shaft. Right, further normalised with $\sqrt{\beta \Delta T}$. Data are averaged in time, in the azimuthal direction and between $x^{*}=0.25$ and 0.75 . 

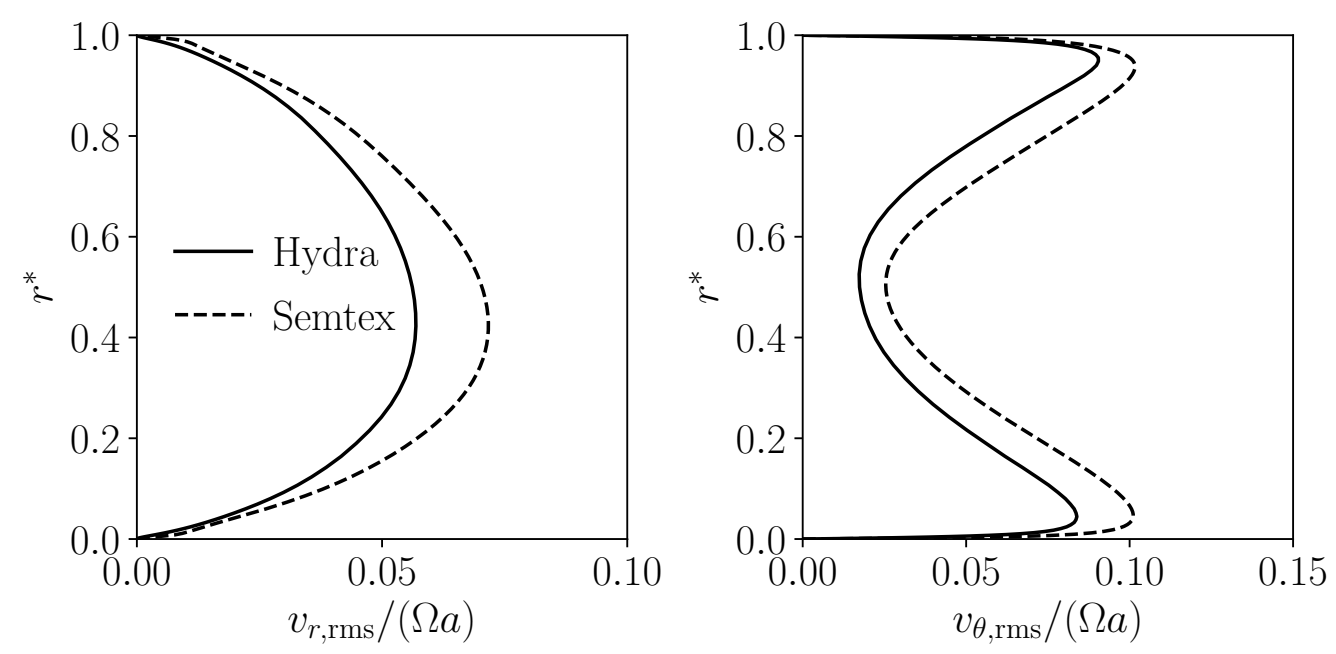

Figure 11: Comparison of radial (left) and azimuthal (right) velocity fluctuation profiles between Hydra and Semtex at $R a=10^{9}$. Data are averaged in time, in the azimuthal direction and between $x^{*}=0.25$ and 0.75 .
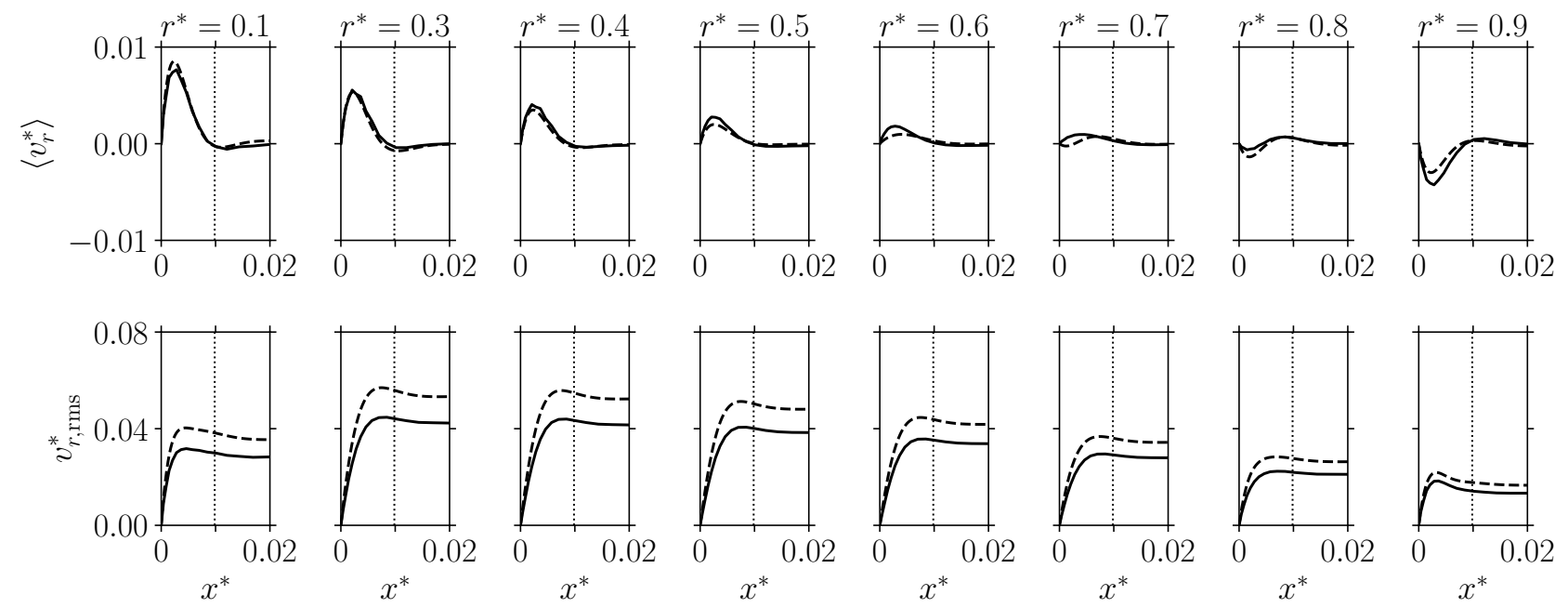

Figure 12: Disc kinetic boundary layer profiles for $R a=10^{9}$. Hydra results: solid lines. Semtex results: dashed lines. Dotted lines indicate the thickness of a laminar Ekman layer, $(\pi / d)(\Omega / \nu)^{-0.5}$ 
The mean radial velocity and its fluctuation profiles are plotted in Fig. 12. It is clear that the disc kinetic boundary layer thickness corresponds well to that for the Ekman layer. Comparing the mean radial velocity profiles between Hydra and Semtex, reasonably good agreement is observed. The near-disc mean radial velocity profiles at low radii show a positive peak, this peak reduces with increasing radius and eventually turns negative. The mean radial velocity outside the disc kinetic boundary layer is close to zero. The radial velocity fluctuations are significantly larger than the mean velocity. Results from Semtex and Hydra are in good agreement apart from the higher level of fluctuations given by Semtex. Similar good agreement is obtained for the tangential velocity component in Fig. 13. However, the difference in $\left\langle v_{\theta}^{*}\right\rangle$ between the two solvers is more pronounced at $r^{*}=0.9$. High peak and far-field mean tangential velocity values are obtained with Hydra. This difference is associated with differences in mean Coriolis force as will be discussed later in Section 5.4.

The difference in velocity fluctuations between the two solvers exists for all the $R a$ values investigated in this paper, but is less marked at lower $R a$. The higher heat transfer in Semtex at $R a=10^{8}$ and $10^{9}$ may contribute to this difference by generating stronger convective plumes. Comparing velocity fluctuations on the different meshes suggests greater sensitivity in Semtex than in Hydra, e.g. the Semtex results show some non-monotonic behaviour (as given in Tab. 3 for the Nusselt number). Thus it is postulated that the different solution schemes may also contribute to these differences.
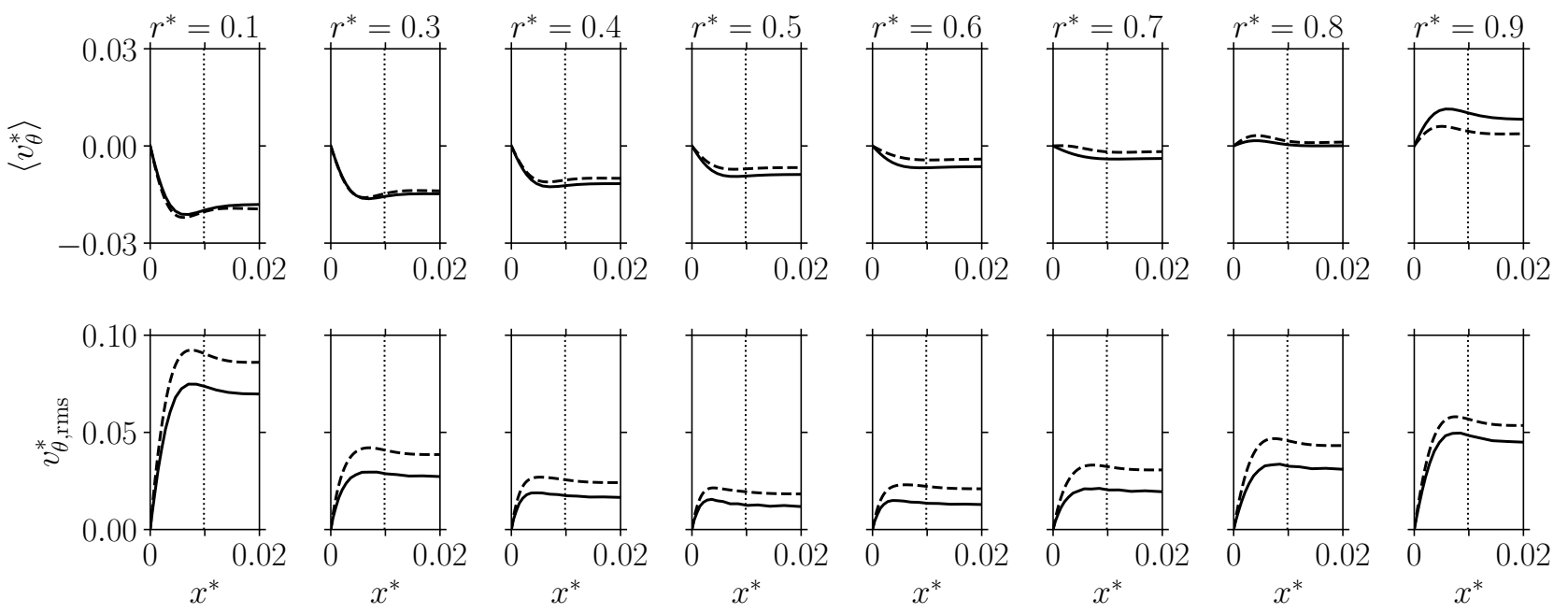

Figure 13: Disc kinetic boundary layer profiles for $R a=10^{9}$. Hydra results: solid lines. Semtex results: dashed lines. Dotted lines indicate the thickness of a laminar Ekman layer, $(\pi / d)(\Omega / \nu)^{-0.5}$.

The scaling of disc kinetic boundary layer thickness is evaluated with the radial velocity fluctuations at $r^{*}=0.5$. As for the shroud thermal boundary layer, the value and position of peak $v_{r, \mathrm{rms}}$ is used to correlate the disc kinetic boundary layer thickness. A good match of the boundary layer at different $R a$ values is shown in the left subplot of Fig. 14 which gives Hydra solutions. The normalised disc boundary layer thicknesses are plotted against $R e_{\phi}$ in the right subplot. Reasonably good agreement is observed with the laminar Ekman layer thickness $(\pi / d)(\Omega / \nu)^{-0.5}$. Note, again, that the determination of the peak location in Hydra solutions relies on the mesh resolution, which gives relatively larger uncertainty for $R a=10^{7}$ due to a thicker boundary layer and expanding mesh. 

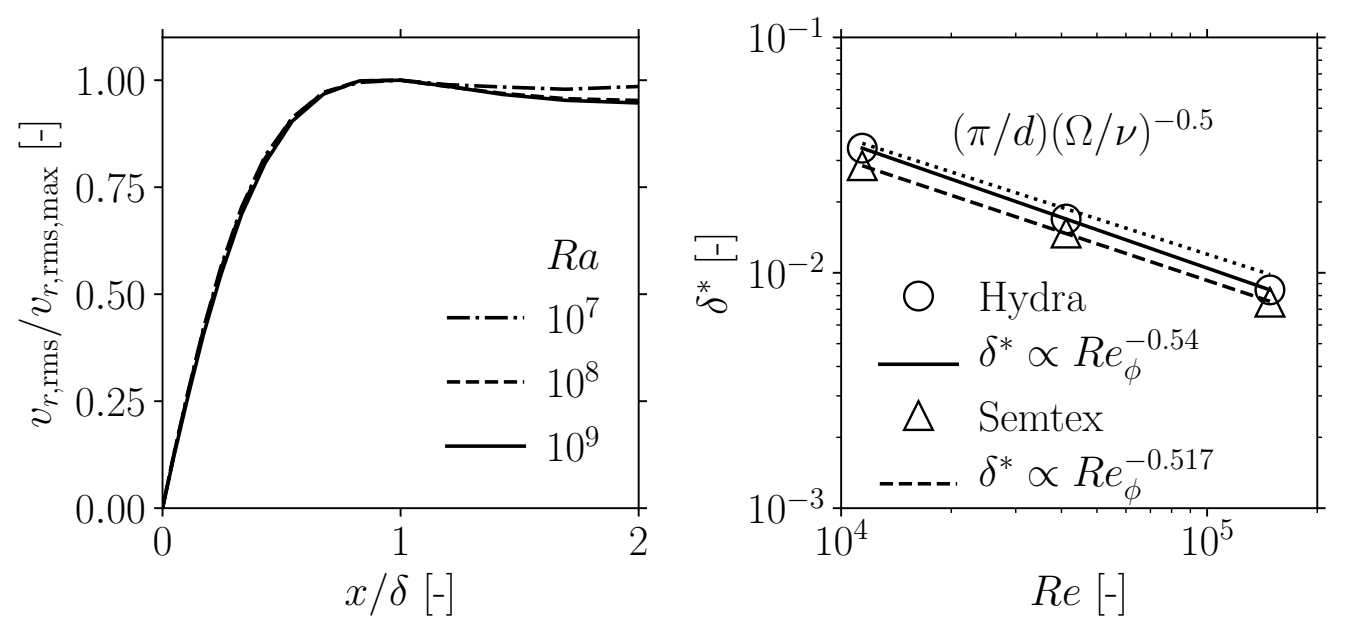

Figure 14: Normalised disc kinetic boundary layer from Hydra's solution (left), and scaling of disc kinetic boundary layer thickness (right) based on $v_{r, \mathrm{rms}}^{*}$. Profiles are extracted at $r^{*}=0.5$. $\delta^{*}=\delta / d$. The dotted line on the right plot corresponds to the laminar Ekman layer thickness, $(\pi / d)(\Omega / \nu)^{-0.5}$.

\subsubsection{Instantaneous velocity profiles}

Statistically the mean radial velocity outside the disc kinetic boundary layer approaches zero, but instantaneously the core has non-zero and non-uniform radial and tangential velocities due to the presence of the counter-rotating cells induced by the buoyancy effect. Pitz et al. [11] presented the laminar Ekman analytical solution for non-zero far-field radial and tangential velocities (as in Eqs. (5) and (6)). They showed, using LES results from the Semtex solver, that the disc boundary layer behaves as a laminar Ekman layer with appropriately selected far-field radial and tangential velocity components.

$$
\begin{aligned}
& v_{r, \mathrm{Ek}}=v_{r, \text { core }}\left(1-e^{-x / \delta_{\mathrm{Ek}}} \cos \frac{x}{\delta_{\mathrm{Ek}}}\right)-v_{\theta, \mathrm{core}} e^{-x / \delta_{\mathrm{Ek}}} \sin \frac{x}{\delta_{\mathrm{Ek}}} \\
& v_{\theta, \mathrm{Ek}}=v_{\theta, \text { core }}\left(1-e^{-x / \delta_{\mathrm{Ek}}} \cos \frac{x}{\delta_{\mathrm{Ek}}}\right)+v_{r, \text { core }} e^{-x / \delta_{\mathrm{Ek}}} \sin \frac{x}{\delta_{\mathrm{Ek}}}
\end{aligned}
$$

Here the Ekman layer solution is compared to Hydra's instantaneous flow field at $R a=10^{9}$, using far-field velocities obtained from their averaged values from the Hydra solution between $x^{*}=0.02$ and 0.03 . The radial and tangential velocity from Hydra and the Ekman layer profiles are plotted together in Fig. 15. The circumferential locations are selected arbitrarily, to show negative and positive radial velocity with high and low values, as well as a near zero radial velocity profile. A simple smoothing function is applied to remove grid-to-grid oscillation of the profiles. Good agreement is achieved between the solutions plotted. This confirms the laminar-Ekman-layer behaviour of the instantaneous disc kinetic boundary layer stands. As stated by Pitz et al. [11] the laminar Ekman layer solution neglects non-linear inertial effects, thus the good agreement indicates much faster response time of the disc boundary layer than the time scale for changes in the far-field velocity. 

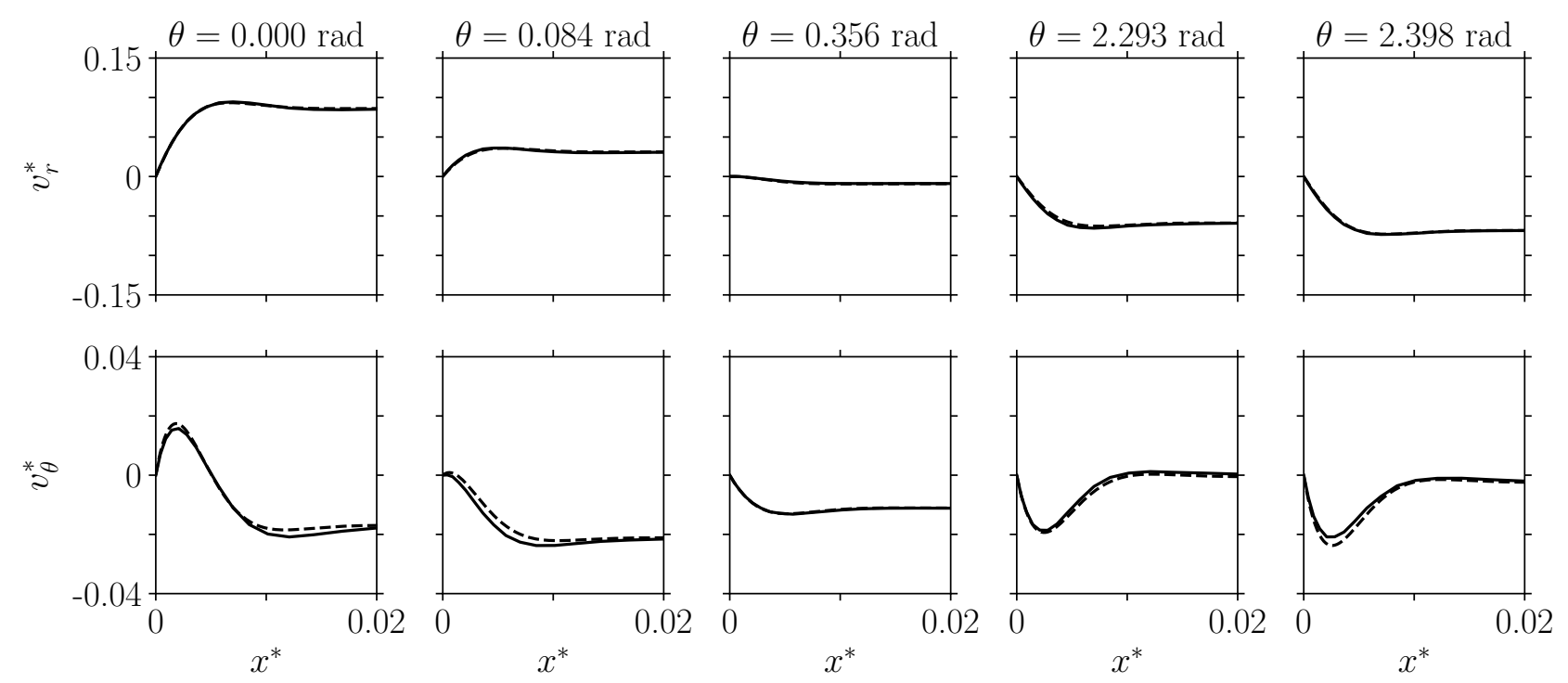

Figure 15: Instantaneous radial and tangential velocity profiles for $R a=10^{9}$, comparison between Hydra (solid curves) and laminar Ekman layer (dashed curves) solutions (presented by Pitz et al. [11]). Core velocities, for Eqs. (5) and (6), are characterised with the averaged values between $x^{*}=0.02$ and 0.03 .

\subsection{Centrifugal and Coriolis forces}

Owen and Long [1] emphasised the role of the Coriolis force in rotating buoyant flows as important in developing cyclonic and anti-cyclonic circulations for transporting heat between the cylindrical surfaces. This force does not appear in natural heat convection between horizontal plates under gravity. As a restoring force the Coriolis force, induced by the relative azimuthal motions of the flow, may act to reduce the radial heat transport induced by the centrifugal buoyancy.

The expression for normalised and averaged centrifugal buoyancy and radial Coriolis force terms from the momentum equation are given in Eqs. (7) and (8) for Hydra and Semtex, respectively. With the Boussinesq approximation, density variation is only considered in the centrifugal force term.

$$
\begin{gathered}
f_{\text {cen }}=\left(\langle\rho\rangle-\left\langle\rho_{\text {core }}\right\rangle\right) \Omega^{2} r /\left(\left\langle\rho_{\text {core }}\right\rangle \Omega^{2} a\right), \quad f_{\text {Cor }}=2\left\langle\rho v_{\theta}\right\rangle \Omega /\left(\left\langle\rho_{\text {core }}\right\rangle \Omega^{2} a\right) \\
f_{\text {cen }}=-\beta\left(\langle T\rangle-\left\langle T_{\text {core }}\right\rangle\right) \Omega^{2} r /\left(\Omega^{2} a\right), \quad f_{\text {Cor }}=2\left\langle v_{\theta}\right\rangle \Omega /\left(\Omega^{2} a\right)
\end{gathered}
$$

Plots of $f_{\text {cen }}$ and $f_{\text {Cor }}$ are shown in the left subplot of Fig. 16 for $R a=10^{9}$. The graph gives a comparison of the forces from Hydra and Semtex. The profiles are averaged in time, the azimuthal direction and between $x^{*}=0.25$ and 0.75 . The centrifugal buoyancy force shows steep gradients across the thermal boundary layers and a negligible core value, reflecting the uniform core temperature. There is a small but noticeable difference between the Hydra and Semtex results near the shroud. The averaged Coriolis force acts in the radially outward direction near the shroud and radially inwards near the shaft, and is clearly significant compared to the centrifugal buoyancy force. Differences between Hydra and Semtex are also significant. By definition the mean Coriolis force is directly associated with the mean tangential velocity, indi- 
cating that the difference in Coriolis force near the shroud may come from the mean tangential velocity. The mean tangential velocity at $r^{*}=0.96$ is plotted in Fig. 17 with both Hydra and Semtex solutions. Note that the left part of the figure is zoomed to show the disc boundary layer. Significant difference in $\left\langle v_{\theta}^{*}\right\rangle$ is shown near the shroud, and extends across the core which is almost invariant, in agreement with the Taylor-Proudman theorem. There might also be an effect of the disc boundary layer to the core.

The averaged centrifugal and Coriolis forces at different $R a$ values are plotted in the righthand side subplot of Fig. 16, using Hydra solutions. A decrease in normalised centrifugal force within the thermal boundary layer is observed as $R a$ increases, due to the reduction of $\beta \Delta T$. The Coriolis force becomes more important with the increase of $R a$. This might be expected from Eqs. (7) and (8), and the observed scaling of velocity with $\Omega a \sqrt{\beta \Delta T}$. The increasingly important Coriolis force could increase the difference of shroud $N u$ in centrifugal buoyant flow from that found in natural heat transfer between horizontal plates under gravity.
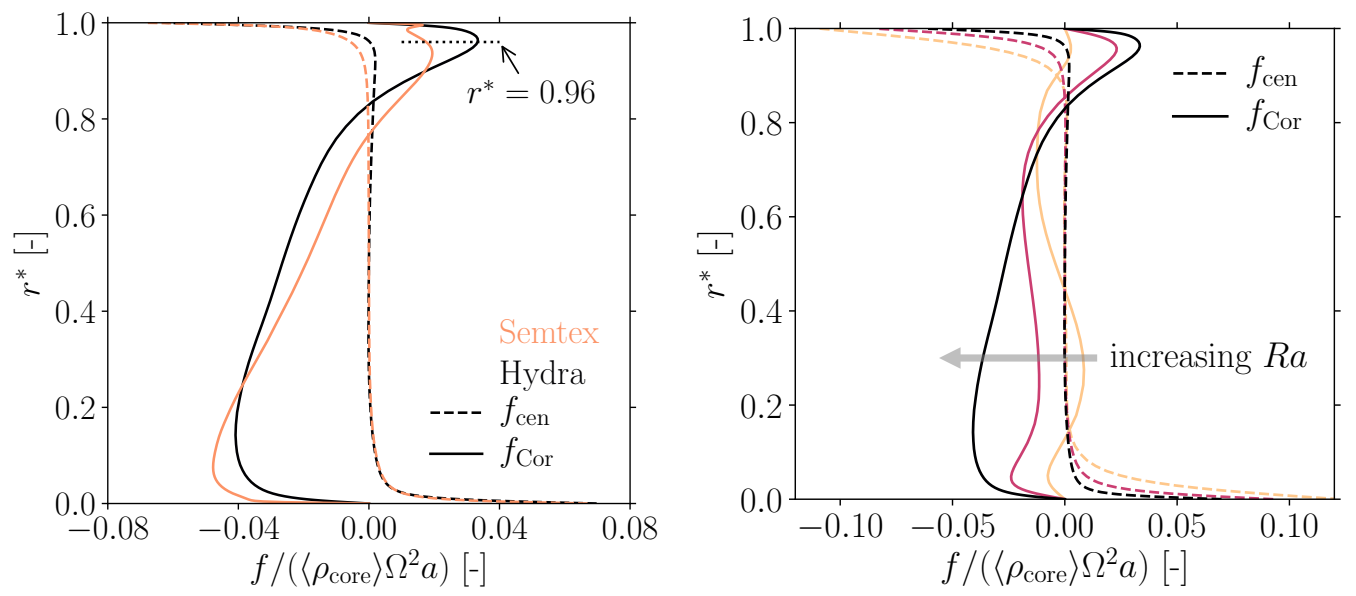

Figure 16: Normalised force terms in the radial momentum equation. Left: comparison between Hydra and Semtex at $R a=10^{9}$; right: Hydra solutions for $R a$ between $10^{7}$ and $10^{9}$. Profiles are averaged in time, the azimuthal direction and between $x^{*}=0.25$ and 0.75 .

\subsection{Ekman layer scrubbing}

In order to investigate further the reason for different shroud $N u$ predictions with the two solvers at $R a=10^{9}$, the mean normalised temperature profile along the horizontal axis is plotted in Fig. 18. This figure plots the temperature profiles from within the shaft boundary layer at $r^{*}=0.0005$ to within the shroud boundary layer $r^{*}=0.9995$. The insets in subplots, at $r^{*}=0.1000$ and 0.9600 , show zoomed views near the disc, confirming the adiabatic boundary condition. Note that in these subplots the vertical axes are different but have the same scale. In the shaft and shroud boundary layers the two solvers show nearly constant temperatures except near the disc wall. Compared with Semtex results, Hydra solutions are closer to the temperature on the cylindrical boundaries, reflecting the difference of $N u$. Moving radially outboard from the shaft, higher near disc temperatures are observed in the Hydra results. In contrast, the temperatures from Semtex are almost uniform apart from very close to the disc. As similar radial velocity profiles are obtained with both solvers as shown in Fig. 12, it is unlikely this 


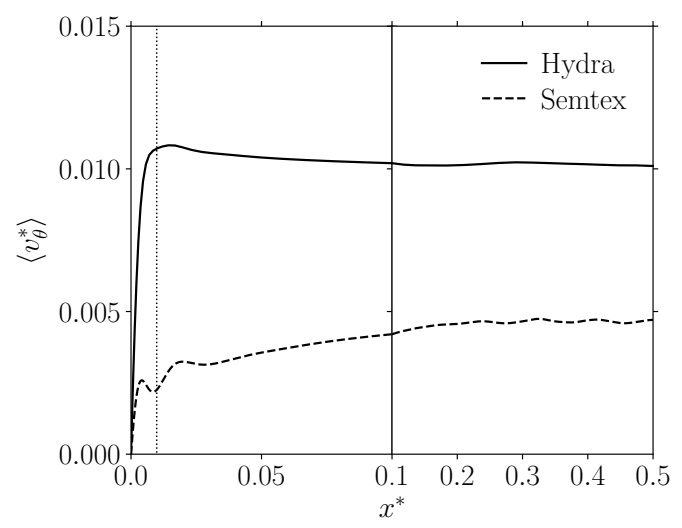

Figure 17: Mean tangential velocity profiles at $r^{*}=0.96$ for $R a=10^{9}$, obtained with Hydra and Semtex. Profiles are averaged in time and the azimuthal direction. Dotted lines indicate the thickness of a laminar Ekman layer, $(\pi / d)(\Omega / \nu)^{-0.5}$. The left part of the figure is zoomed to show the disc boundary layer.

different is caused by advection. An explanation for the near disc high temperature in Hydra, despite the small Eckert number $(\sim 1 \%)$, is viscous energy dissipation. As shown in Figs. 12 and 13 the mean velocities are very small. In the absence of the mean convection, viscous dissipation of the kinetic energy in the fluctuating boundary layers leads to an accumulation of internal energy. The greater strength of the velocity fluctuations, compared to the mean velocity, and the thin shear layer induced by the Coriolis force contribute to this effect. This kinetic energy generated by buoyancy is converted to internal energy by the "Ekman layer scrubbing".

A crossover in temperature level between the two solvers is seen at $r^{*}=0.96$, where Hydra's core temperature is lower than Semtex's. This might be associated with the radial Coriolis force caused by circumferential velocity as shown in Fig. 17. The higher Coriolis force in Hydra prevents the hot fluid from moving radially inboard, leading to a lower core temperature.

The Hydra solution at $R a=10^{8}$ is also plotted in the figure. Comparing the Hydra results between $R a=10^{8}$ and $10^{9}$, the Ekman layer scrubbing effect is weaker at low Eckert number $\left(R a=10^{8}\right)$.

The significance of the discs is reinforced by comparison with pseudo-2D test cases where the discs are replaced with periodic boundary conditions. The Ekman layer scrubbing and other disc effects are eliminated, and the difference in shroud $N u$ between the two solvers reduces to within $10 \%$ for $R a=10^{9}[27]$.

\subsection{Turbulent kinetic energy budget}

In turbulent flows, the turbulent kinetic energy budget can show how the turbulent kinetic energy (TKE) is generated, transported and dissipated. In a typical turbulent boundary layer TKE is generated away from the wall, transferred towards the wall through transport terms and eventually dissipated close to the wall. The TKE budget has been studied in RB convection between horizontal plates under gravity, with and without rotation about the vertical axis $[28,29]$. Different mechanisms from the typical turbulent boundary layer are observed. It is also of interest to know the TKE budget balance behaviour in the present purely centrifugal 

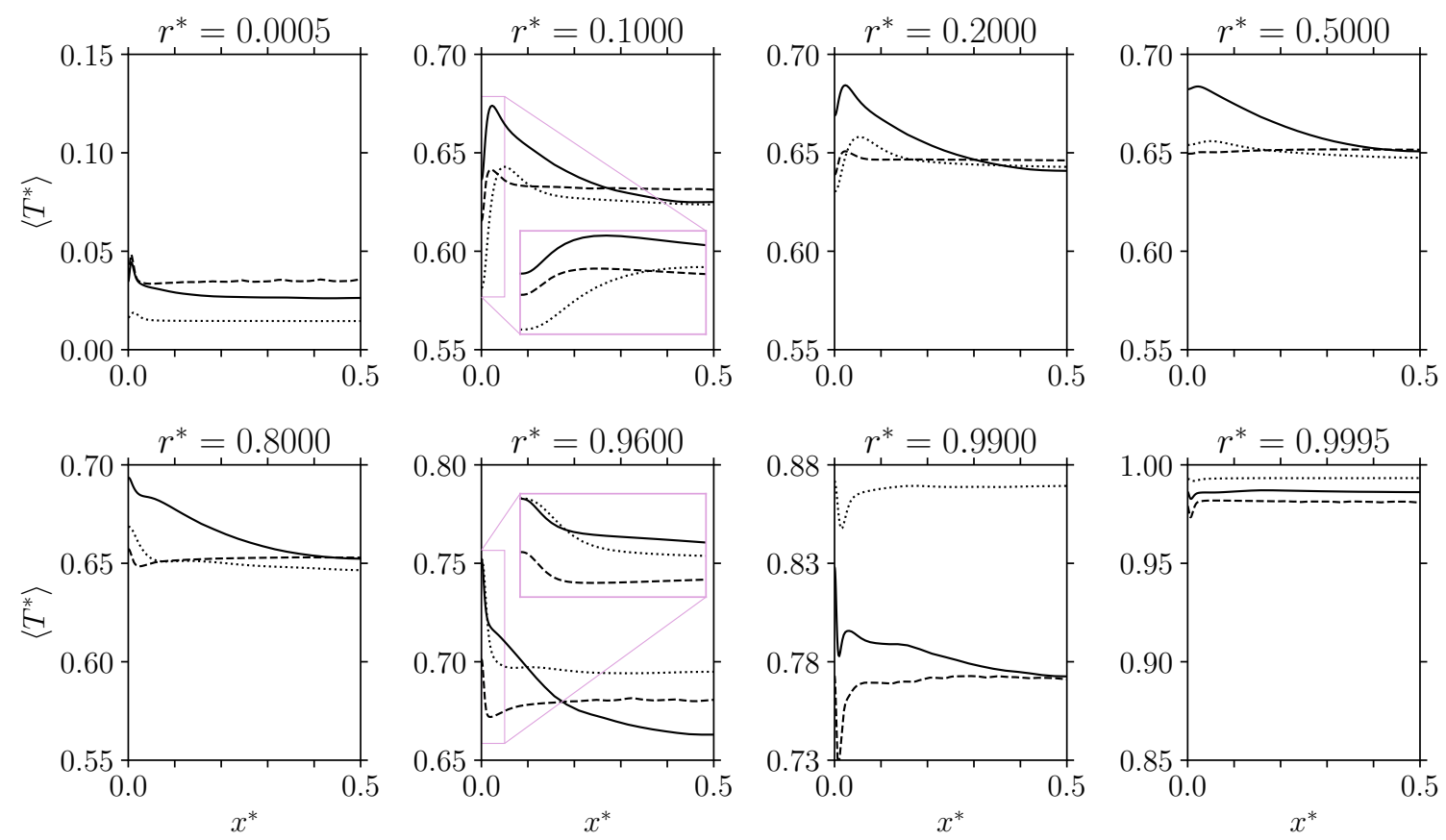

Figure 18: Normalised mean temperature profiles along the axial direction, at different radii. Hydra results at $R a=10^{9}$ : solid lines. Semtex results at $R a=10^{9}$ : dashed lines. Hydra results at $R a=10^{8}$ : dotted lines. Insets in subplots at $r^{*}=0.1000$ and 0.9600 show zoomed views near the disc. Data are averaged in time and the circumferential direction. Note that the vertical axis scales are different but have the same scale. 
buoyancy-induced flows. The TKE budget equation for centrifugal buoyant flows is given in Appendix B, in which only a buoyancy production term emerges in addition to the standard TKE budget equation. According to Pitz et al. [10] the flow state enters turbulent regimes for $R a$ above $10^{8}$. Note, however, that the axial variation of the flow is restricted by the Taylor-Proudman theorem, indicating two-component turbulence in the core. The $-5 / 3$ slope of velocity spectrum expected for turbulent flows was also observed numerically by Sun et al. [3] for their studies at $R a=10^{9}$ on the same configuration.

The TKE budget for the test case at $R a=10^{9}$, obtained with Hydra, is illustrated in the left subplot of Fig. 19, in which the transport term is a combination of other terms not plotted on the graph. Note that the averaging is realised in time and the azimuthal direction. A pronounced buoyancy term is seen in the core dominating the production of the TKE. It is also noted that the buoyancy production is constant in the core. Production due to flow shear is smaller and negative values are shown within the shroud and shaft boundary layers. Typical effects of near wall dissipation are observed. However, in the core the dissipation does not vanish. A similar phenomenon is reported by Kunnen et al. [29], where the dissipation predominately balances the buoyancy production. Transport of turbulence is an important effect here, and is more significant than in Kunnen et al.'s results. In Kunnen et al.'s configuration the gravitational acceleration is significantly smaller than the centripetal acceleration. This large buoyancy production in the present case is balanced by the terms containing the reduced pressure in which the constant part of the centrifugal buoyancy term is absorbed.
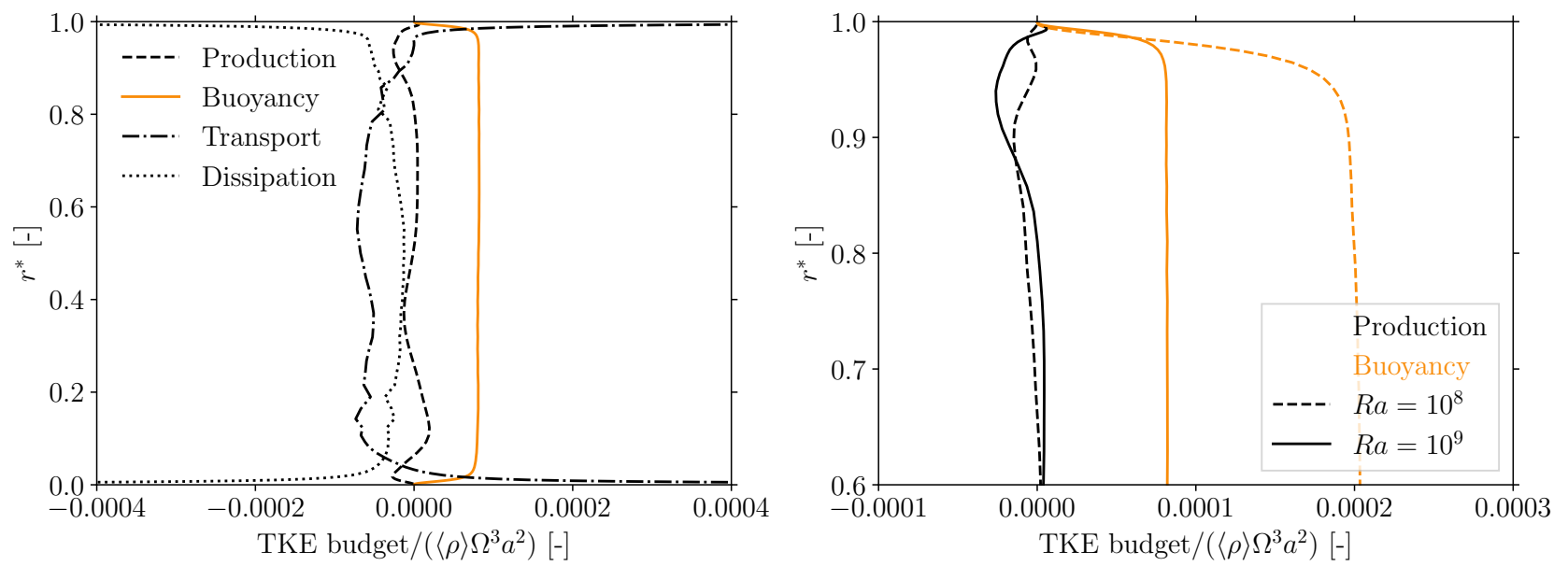

Figure 19: Turbulent kinetic energy budget for $R a=10^{9}$ (left) and comparison between $R a=$ $10^{8}$ and $10^{9}$ (right), at the mid-axial position. Data are averaged in time and the azimuthal direction.

A comparison of the production and buoyancy terms between $R a=10^{8}$ and $10^{9}$ is shown in the right-hand side subplot of Fig. 19, at the shroud. As $R a$ is increased the buoyancy production term reduces, due to the decrease of $\beta \Delta T$ following the operating condition of Bohn et al. [7]. The production due to flow shear is negligible in the bulk, and the negative production is greater at higher $R a$ value. 


\section{$6 \quad$ Further discussion}

Two widely recognised simplifications are employed in the use of an incompressible solver to investigate buoyancy-induced flows. The first one is the Boussinesq approximation which uses the buoyancy parameter $\beta \Delta T$ to account for the effect of density variation on the buoyancy generating term, i.e. the centrifugal buoyancy term. The limit of using this approximation is believed to be $\beta \Delta T \lesssim 0.2$ for heat transfer between horizontal plates under gravity. In the test cases presented in the present study the maximum $\beta \Delta T$ is considered up to $0.174\left(R a=10^{7}\right)$, and only $3.2 \%$ difference in $N u$ is found between the two solvers.

The second simplification is to use the temperature transport equation as the energy equation. This requires the Eckert number to be sufficient small to neglect the effects of pressure change, the kinetic energy, the viscous term and the body forces. For the conditions considered in this work the maximum $E c$ is 0.013 , which is far smaller than those generally seen in real engines. Despite this, the viscous effects near the discs associated with fluctuating Ekman layers is non-negligible at $R a=10^{9}$.

Regarding the computational demand, for example at $R a=10^{9}$, Hydra has consumed $\sim 300$ hours wall time with 2400 CPU cores (E5-2697 v2), while Semtex took $~ 96$ hours with 256 CPU cores (E5-2660 v4). Assuming Moore's law holds, full compressible LES is still far from possible to be used for engine design.

\section{Concluding remarks}

The buoyancy-induced flow in a simple sealed rotating cavity has been investigated. DNS results from an incompressible Navier-Stokes equation solver, Semtex, based on the Boussinseq approximation are compared with LES from a fully compressible gas flow code Hydra. Reasonably good agreement between the two solvers is achieved for the operating conditions considered $\left(R a \in\left[10^{7}, 10^{9}\right]\right)$. The main conclusions of the present study are:

- While there is much general agreement between the two models, significant difference in overall heat transfer prediction has been found. With the Hydra solutions good agreement with the original experimental correlation is achieved for $R a=10^{7}$ but the difference increases to around $20 \%$ at $R a=10^{9}$ where a corrected experimental correlation is used. Experimental uncertainty may contribute to this difference. Semtex predictions with the Boussinesq approximation show good agreement with Hydra at $R a=10^{7}$ but diverge from the Hydra results and experimental correlations as $R a$ increases.

- Hydra predictions of shroud $N u$ show a scaling of $N u \propto R a^{0.286}$, in close agreement with the corrected experimental correlation provided by Bohn and Gier [14] and the $R a^{2 / 7}$ scaling in $\mathrm{RB}$ convection for the $R a$ and $P r$ range in the present work [19]. Semtex presents a scaling of $N u \propto R a^{1 / 3}$, consistent with the $N u$ scaling for heat transfer between horizontal plates under gravity at high $R a$ number provided by Hollands et al. [17].

- A mesh resolution issue on the averaged core temperature is found with Semtex in comparison with the results of Pitz et al. [11]. The DNS results presented in the present study show normalised mean core temperature decreasing slightly with the increase of $R a$, whereas Pitz et al.'s LES results showed the opposite trend. Interestingly, in Hydra 
solutions the normalised mean core temperature stays the same regardless of $R a$ values for the conditions considered.

- Agreeing with RB convection [19], the shroud thermal boundary layer thickness can be estimated with $\lambda^{*}=0.5 N u^{-1}$ in both solvers. The velocity $\Omega a \sqrt{\beta \Delta T}$ appears to provide a useful scaling to correlate the shroud kinetic boundary layer between different Rayleigh numbers.

- The solutions from Hydra confirm both the mean and instantaneous laminar Ekman layer behaviour within the disc kinetic boundary layer reported by Pitz et al. [11] with Semtex. This is also valid for $R a=10^{9}$.

- Viscous dissipation of kinetic energy associated with the Ekman layer scrubbing is considered mainly responsible for the difference in mean shroud heat transfer between the two solvers.

- The turbulent kinetic energy budget is dominated by a constant buoyancy production term in the core.

- The maximum $\beta \Delta T$ considered in this study is $0.174\left(R a=10^{7}\right)$ at which condition the best agreement of shroud $N u$ is achieved, suggesting that the use of the Boussinesq approximation at this level of $\beta \Delta T$ is appropriate for centrifugal buoyant flows. However, by analogy with gravitational buoyancy, loss of accuracy of the Boussinesq approximation is expected for $\beta \Delta T>0.2$ and further attention to the Eckert number effects is needed in considering applications at engine conditions.

\section{Nomenclature}

\section{Greek Symbols}

\section{Symbol Description}

$\alpha$

$\beta$

$\beta \Delta T$

$\delta$

$\Delta()_{\max }$

$\delta^{*}$

$\delta_{\mathrm{Ek}}$ thickness

Ekman depth
Thermal diffusivity

Thermal expansion coefficient

Buoyancy parameter
Expressions or Values

$$
\begin{aligned}
\alpha & =\frac{\kappa}{\rho C_{p}} \\
\beta & =1 / T_{m}
\end{aligned}
$$$$
K^{-1}
$$

$\mathrm{m}$

Disc kinetic boundary lay
on the maximum $v_{r, \text { rms }}$

Maximum grid spacing

$\mathrm{mm}$

Normalised disc kinetic boundary layer $\quad \delta^{*}=\delta / d$

$$
\delta_{\mathrm{Ek}}=(\Omega / \nu)^{-0.5}
$$


$\begin{array}{llll}\Delta_{w} & \text { Near wall grid spacing } & - & \mathrm{mm}\end{array}$

$\Delta T \quad$ Temperature difference between shroud and $\Delta T=T_{b}-T_{a} \quad \mathrm{~K}$ shaft

$\kappa \quad$ Thermal conductivity

$\mathrm{W} \cdot \mathrm{m}^{-1} \cdot \mathrm{K}^{-1}$

$\lambda \quad$ Shroud thermal boundary layer thickness -

$\mathrm{m}$ based on the maximum $T_{\text {rms }}$

$\lambda^{*} \quad$ Normalised shroud thermal boundary layer $\lambda^{*}=\lambda /(b-a)$ thickness

$\mu \quad$ Dynamic viscosity

$\mathrm{kg} \cdot \mathrm{m}^{-1} \cdot \mathrm{s}^{-1}$

Kinematic viscosity

$\nu=\mu / \rho$

$\mathrm{m}^{2} \cdot \mathrm{s}^{-1}$

Angular speed of rotor

$\operatorname{rad} \cdot \mathrm{s}^{-1}$

Density

$\mathrm{kg} \cdot \mathrm{m}^{-3}$

$\rho$

Numerical dissipation

See Eq. (19)

$\varepsilon_{\text {num }}$

Subgrid scale dissipation

See Eq. (19)

$\varepsilon_{\mathrm{SGS}}$

Total dissipation

See Eq. (19)

$\mathrm{kg} \cdot \mathrm{m}^{-1} \cdot \mathrm{s}^{-3}$

$\mathrm{kg} \cdot \mathrm{m}^{-1} \cdot \mathrm{s}^{-3}$

$\mathrm{kg} \cdot \mathrm{m}^{-1} \cdot \mathrm{s}^{-3}$

\section{Roman Symbols}

Symbol Description

a Shaft radius

$b \quad$ Shroud radius

$C_{p}$

$d$

$E c$

$N_{e l}$

$N_{t o t}$

$N_{x, r, \theta}$

$P$

$\operatorname{Pr}$

Eckert number

Prandtl number

$N u \quad$ Shroud Nusselt number. Ratio of convective $N u=q_{\text {conv }} / q_{\text {cond }}$

Specific heat capacity at constant pressure. $C_{p}=1004.15$

$a=0.125$

$b=0.24$

$d=0.12$

$$
E c=\frac{\Omega^{2} r_{m}^{2}}{C_{p} \Delta T}
$$

\section{Units}

$\mathrm{m}$

m

$\mathrm{J} \cdot \mathrm{kg}^{-1} \cdot \mathrm{K}^{-1}$

$\mathrm{m}$

Number of elements in a meridional plane.

Total number of grid points.

Number of grid points in the axial, radial and tangential directions. heat flux to conductive heat flux.

Polynomial order, or reduced pressure.

$$
\operatorname{Pr}=\frac{\nu}{\alpha}=0.7
$$




$$
r^{*}=r /(b-a)
$$

$R a \quad$ Rayleigh number $R a=\frac{\rho^{2} \Omega^{2} r_{m} \beta \Delta T(b-a)^{3}}{\mu^{2}} \operatorname{Pr}$

$R e_{\phi} \quad$ Rotational Reynolds number $R e_{\phi}=\frac{\rho \Omega r_{m}(b-a)}{\mu}$

$T^{*} \quad$ Normalised static temperature

$$
T^{*}=\left(T-T_{a}\right) / \Delta T
$$

$v_{\theta}^{*} \quad$ Normalised azimuthal velocity

$v_{\theta}^{*}=v_{\theta} /(\Omega r)$

Normalised radial velocity

$v_{r}^{*}=v_{r} /(\Omega r)$

Normalised axial position.

$$
x^{*}=x / d
$$

\section{Subscripts}

\section{Symbol Description}

\begin{tabular}{|c|c|}
\hline $\mathrm{a}$ & Shaft \\
\hline $\mathrm{b}$ & Shroud \\
\hline cen & Centrifugal \\
\hline Cor & Coriolis \\
\hline core & Core value \\
\hline $\mathrm{m}$ & $\begin{array}{l}\text { Arithmetic mean of the values on the } \\
\text { shroud and shaft }\end{array}$ \\
\hline $\max$ & Maximum value \\
\hline rms & Root mean square \\
\hline
\end{tabular}

\section{Other Symbols}

\section{Symbol}

$\langle\cdot\rangle$

\section{Description}

Ensemble average 


\section{Appendix}

\section{A Governing equations}

Both the flow solvers - Hydra and Semtex - used in this study solve the Navier-Stokes equations. Hydra considers the fully compressible form for gas flow, whereas Semtex employs the incompressible form. This section compares the two equation forms aiming to reveal their differences, both in terms of the Boussinesq approximation, used to account for density variations, and in the form of the energy equation..

\section{A.1 Compressible Navier-Stokes equations}

The flow field is solved in a relative frame of reference rotating at a constant angular speed about the axis. The continuity and momentum equations may be written,

$$
\begin{gathered}
\frac{\partial \rho}{\partial t}+\vec{\nabla} \cdot(\rho \vec{u})=0 \\
\frac{\partial \rho \vec{u}}{\partial t}+\vec{\nabla} \cdot(\rho \vec{u} \otimes \vec{u})=-\vec{\nabla} p+\mu\left[\vec{\nabla}^{2} \vec{u}+\frac{1}{3} \vec{\nabla}(\vec{\nabla} \cdot \vec{u})\right]-2 \rho \vec{\Omega} \times \vec{u}-\rho \vec{\Omega} \times(\vec{\Omega} \times \vec{r})
\end{gathered}
$$

where $\otimes$ denotes the outer product, i.e. $\vec{u} \otimes \vec{u}=\vec{u} \vec{u}^{\top}$. The terms $-2 \rho \vec{\Omega} \times \vec{u}$ and $-\rho \vec{\Omega} \times(\vec{\Omega} \times$ $\vec{r})$ represent, respectively, the Coriolis and centrifugal force terms. Generally, to clarify the buoyancy generating term, the constant density part of the centrifugal term, $\langle\rho\rangle \vec{\Omega} \times(\vec{\Omega} \times \vec{r})$, is absorbed into the pressure gradient forming what is termed reduced pressure $(P)$. Note that $\langle\rho\rangle$ represents the locally averaged density. Therefore, the compressible momentum equation in a rotating frame of reference can be expressed as,

$$
\frac{\partial \rho \vec{u}}{\partial t}+\vec{\nabla} \cdot(\rho \vec{u} \otimes \vec{u})=-\vec{\nabla} P+\mu\left[\vec{\nabla}^{2} \vec{u}+\frac{1}{3} \vec{\nabla}(\vec{\nabla} \cdot \vec{u})\right] \underbrace{-2 \rho \vec{\Omega} \times \vec{u}}_{\text {Coriolis force term. Buoyancy generating term. }} \underbrace{-\rho^{\prime} \vec{\Omega} \times(\vec{\Omega} \times \vec{r})}
$$

with $\rho^{\prime}=\rho-\langle\rho\rangle$ and $P=p+\frac{1}{2}\langle\rho\rangle(\vec{\Omega} \times \vec{r})^{2}$.

The energy equation is solved in its relative form in Hydra, $\rho E_{r}=\rho e+\frac{1}{2} \rho \vec{u}^{2}-\frac{1}{2} \rho(\vec{\Omega} \times \vec{r})^{2}$. Considering rothalpy $I=E_{r}+p / \rho$ as the convection term, the work by the centrifugal force is then eliminated in the source terms of the energy equation,

$$
\frac{\partial \rho E_{r}}{\partial t}+\vec{\nabla} \cdot(\rho \vec{u} I)=\vec{\nabla} \cdot(\kappa \vec{\nabla} T)+\vec{\nabla} \cdot(\tau \cdot \vec{u})
$$

with $\kappa=\mu C_{p} / \operatorname{Pr}$ the thermal conductivity, and $\tau$ the viscous stress tensor. 


\section{A.2 Incompressible Navier-Stokes equations}

The incompressible form of the Navier-Stokes equations is considered in Semtex. Assuming a constant density $\rho=\rho_{0}$, the mass conservation law in Eq. (9) reduces to,

$$
\vec{\nabla} \cdot \vec{u}=0
$$

Imposing a constant density $\rho=\rho_{0}$ in all terms apart from the buoyancy generating term, the momentum equation in rotating frame from Eq. 11 can be expressed as,

$$
\rho_{0} \frac{\partial \vec{u}}{\partial t}+\rho_{0} \vec{u} \cdot \vec{\nabla} \vec{u}=-\vec{\nabla} P+\rho_{0} \nu \vec{\nabla}^{2} \vec{u} \underbrace{-2 \rho_{0} \vec{\Omega} \times \vec{u}}_{\text {Coriolis force term. Buoyancy generating term. }} \underbrace{-\rho^{\prime} \vec{\Omega} \times(\vec{\Omega} \times \vec{r})}
$$

This is directly analogous to the Boussinesq approximation in gravitational fields, where the term $-\rho^{\prime} \vec{g}$ is responsible for providing the buoyancy force, and $\vec{g}$ is the gravitational acceleration vector. The effect of density variations is modelled by the approximation,

$$
-\rho^{\prime}=\rho_{0}-\rho=\rho_{0} \beta\left(T-T_{0}\right)
$$

where $\beta$ is the thermal expansion coefficient, usually defined by $\beta=1 / T_{\infty}$ for a gas flow. In the study of centrifugal buoyancy by Bohn et al., $T_{\infty}$ is specified as the arithmetic mean of the temperatures on the shroud and shaft, $T_{\infty}=T_{m}=\frac{1}{2}\left(T_{a}+T_{b}\right) . T_{0}$ is the reference static temperature.

Three conditions should be satisfied for the Boussinsesq approximation to be valid [30]: (a) the density variations $\rho^{\prime}$ are relatively small compared to the reference density, $\rho^{\prime} \ll \rho_{0}$, e.g. $\rho^{\prime} \lesssim 0.2 \rho$; (b) density is a function of temperature only, i.e. $\rho=\rho(T)$; (c) density variations are only essential in potential terms, i.e. those that are able to be expressed as the gradient of a scalar.

Taking the above formulation, Pitz [30] applied the Boussinesq approximation to the centrifugal buoyancy term, which was considered as the only potential term. Then Eq. (14) can be written as,

$$
\frac{\partial \vec{u}}{\partial t}+\vec{u} \cdot \vec{\nabla} \vec{u}=-\frac{\vec{\nabla} P}{\rho_{0}}+\nu \vec{\nabla}^{2} \vec{u} \underbrace{-2 \vec{\Omega} \times \vec{u}}_{\text {Coriolis force term. }}+\underbrace{\beta\left(T-T_{0}\right) \vec{\Omega} \times(\vec{\Omega} \times \vec{r})}_{\text {Buoyancy generating term. }}
$$

The relation between the pressure and velocity fields is solved using a Poisson equation for the pressure.

Some assumptions are made in deriving the incompressible energy equation to provide the temperature field required by the Boussinesq approximation. First, it is commonly accepted that, if the Eckert number $E c=\Omega^{2} r_{m}^{2} /\left(C_{p} \Delta T\right)$ is sufficiently small $(E c \ll 1)$, the effect of pressure change, the kinetic energy, the viscous term and the body force terms can be neglected. Equation (12) can be simplified as,

$$
\frac{\partial C_{v} T}{\partial t}+\vec{u} \cdot\left(C_{p} T\right)=\alpha \vec{\nabla}^{2} C_{p} T
$$

with $\alpha=\kappa /\left(\rho C_{p}\right)$ the thermal diffusivity.

Second, for an incompressible fluid the heat capacities at constant pressure $C_{p}$ and at constant volume $C_{v}$ are identical. Thus, the incompressible energy equation can be further 
reduced as a transport equation of temperature, as implemented in Semtex [31],

$$
\frac{\partial T}{\partial t}+\vec{u} \cdot \vec{\nabla} T=\alpha \vec{\nabla}^{2} T
$$

Conditions for validity of this approach for a compressible fluid in gravitational convection have been considered by Spiegel and Veronis [32]. These conditions appear to be satisfied for the present study.

\section{B Turbulent kinetic energy budget equation}

For centrifugal buoyant flows, a buoyancy generating term $(\bar{\rho}-\langle\bar{\rho}\rangle) \Omega^{2} r$ emerges in the radial momentum equation, with the constant centrifugal force $\langle\bar{\rho}\rangle \Omega^{2} r$ being absorbed into the pressure term forming the reduced pressure $\left(\bar{P}=\bar{p}+\frac{1}{2}\langle\bar{\rho}\rangle(\Omega r)^{2}\right)$. As expressed in Eq. (19), only one additional term emerges accounting for the buoyancy production in the standard TKE budget equation.

$$
\begin{aligned}
\frac{\partial\langle\bar{\rho} k\rangle}{\partial t}=0= & \underbrace{-\frac{\partial}{\partial x_{j}}\left(\langle\bar{\rho} k\rangle\left[\tilde{u}_{j}\right]\right.}_{\begin{array}{c}
\text { mean flow } \\
\text { advection }
\end{array}}) \underbrace{-\left\langle\bar{\rho} u_{i}^{\prime \prime} u_{j}^{\prime \prime}\right\rangle \frac{\partial\left[\tilde{u}_{i}\right]}{\partial x_{j}}}_{\text {production }} \underbrace{-\langle\bar{\rho}\rangle \Omega^{2} r\left\langle v_{r}^{\prime \prime}\right\rangle}_{\begin{array}{c}
\text { buoyancy } \\
\text { production }
\end{array}} \underbrace{-\frac{\partial}{\partial x_{j}}\left\langle\bar{\rho} u_{j}^{\prime \prime} k\right\rangle}_{\begin{array}{c}
\text { turbulent } \\
\text { diffusion }
\end{array}} \underbrace{-\frac{\partial}{\partial x_{i}}\left\langle u_{i}^{\prime \prime} P^{\prime}\right\rangle}_{\begin{array}{c}
\text { pressure } \\
\text { diffusion }
\end{array}}+\underbrace{\left\langle P^{\prime} \frac{\partial u_{i}^{\prime \prime}}{\partial x_{i}}\right\rangle}_{\begin{array}{c}
\text { pressure } \\
\text { dilatation }
\end{array}} \\
& \underbrace{-\left\langle u_{i}^{\prime \prime}\right\rangle \frac{\partial\langle\bar{P}\rangle}{\partial x_{i}}}_{\begin{array}{c}
\text { flux } \\
\text { variation }
\end{array}}-\underbrace{\left\langle\overline{\tau_{i j}} \frac{\partial u_{i}^{\prime \prime}}{\partial x_{j}}\right\rangle}_{\text {dissipation }} \underbrace{+\frac{\partial}{\partial x_{j}}\left\langle u_{i}^{\prime \prime} \overline{\tau_{i j}}\right\rangle}_{\begin{array}{c}
\text { viscous } \\
\text { diffusion }
\end{array}} \underbrace{-\left\langle\overline{\Pi_{i j}} \frac{\partial u_{i}^{\prime \prime}}{\partial x_{j}}\right\rangle}_{\begin{array}{c}
\text { SGS } \\
\text { dissipation }
\end{array}} \underbrace{\frac{\partial}{\partial x_{j}}\left\langle u_{i}^{\prime \prime} \overline{\Pi_{i j}}\right\rangle}_{\begin{array}{c}
\text { SGS } \\
\text { diffusion }
\end{array}} \underbrace{+\Xi_{i i}}_{\begin{array}{c}
\text { Numerical } \\
\text { dissipation }
\end{array}}
\end{aligned}
$$

In this equation a filtered quantity $q$ is denoted by $\bar{q}$. The Favre filter of a quantity $q$ is $\widetilde{q}=\overline{\rho q} / \bar{\rho}$. The ensemble average of a quantity $q$ is described as $\langle q\rangle$. The Favre ensemble averaged of a quantity $q$ is shown as $[q]=\langle\rho q\rangle /\langle\rho\rangle$. Furthermore, the fluctuation of a filtered quantity is indicated by $q^{\prime}=\bar{q}-\langle\bar{q}\rangle$. The Favre fluctuation of a Favre filtered quantity is noted as $q^{\prime \prime}=\widetilde{q}-[\widetilde{q}]$. The term $\left\langle v_{r}^{\prime \prime}\right\rangle$ in the buoyancy production term can be derived with $\left\langle v_{r}^{\prime \prime}\right\rangle=\left\langle\widetilde{v_{r}}\right\rangle-\left[\widetilde{v_{r}}\right]$.

\section{Acknowledgements}

The authors are grateful for the financial support provided by the Engineering and Physical Sciences Research Council under grant EP/P004091, and thank colleagues at the University of Surrey, the University of Bath and Rolls-Royce plc for their technical support. The computing resources are provided by ARCHER and local clusters in the University of Surrey.

\section{References}

[1] J. M. Owen and C. A. Long. Review of buoyancy-induced flow in rotating cavities. Journal of Turbomachinery, 137(11):111001, 2015. 
[2] D. B. Pitz, J. W. Chew, O. Marxen, and N. J. Hills. Direct numerical simulation of rotating cavity flows using a spectral element-Fourier method. Journal of Engineering for Gas Turbines and Power, 139(7):072602, 2017.

[3] Z. Sun, A. Kilfoil, J. W. Chew, and N. J. Hills. Numerical simulation of natural convection in stationary and rotating cavities. In Proceedings of ASME Turbo Expo 2004: Power for Land, Sea, and Air, 2004. Paper No. GT2004-53528.

[4] P. R. Farthing, C. A. Long, J. M. Owen, and J. R. Pincombe. Rotating cavity with axial throughflow of cooling air: flow structure. Journal of Turbomachinery, 114(1):237-246, 1992.

[5] N. R. Atkins and V. Kanjirakkad. Flow in a rotating cavity with axial throughflow at engine representative conditions. In Proceedings of ASME Turbo Expo 2014: Turbomachinery Technical Conference and Exposition, 2014. Paper No. GT2014-27174.

[6] Z. Sun, D. Amirante, J. W. Chew, and N. J. Hills. Coupled aerothermal modeling of a rotating cavity with radial inflow. Journal of Engineering for Gas Turbines and Power, 138(3):032505, 2016.

[7] D. Bohn, E. Deuker, R. Emunds, and V. Gorzelitz. Experimental and theoretical investigations of heat transfer in closed gas-filled rotating annuli. Journal of Turbomachinery, 117(1):175-183, 1995.

[8] M. R. Puttock-Brown, M. G. Rose, and C. A. Long. Experimental and computational investigation of Rayleigh-Bénard flow in the rotating cavities of a core compressor. In Proceedings of ASME Turbo Expo 2017: Turbomachinery Technical Conference and Exposition, 2017. Paper No. GT2017-64884.

[9] M. R. Puttock-Brown and M. G. Rose. Formation and evolution of Rayleigh-Bénard streaks in rotating cavities. In Proceedings of ASME Turbo Expo 2018: Turbomachinery Technical Conference and Exposition, 2018. Paper No. GT2018-75497.

[10] D. B. Pitz, O. Marxen, and J. W. Chew. Onset of convection induced by centrifugal buoyancy in a rotating cavity. Journal of Fluid Mechanics, 826:484-502, 2017.

[11] D. B. Pitz, J. W. Chew, and O. Marxen. Large-eddy simulation of buoyancy-induced flow in a sealed rotating cavity. Journal of Engineering for Gas Turbines and Power, 141(2):021020, 2018.

[12] H. Tang and J. M. Owen. Theoretical model of buoyancy-induced heat transfer in closed compressor rotors. Journal of Engineering for Gas Turbines and Power, 140(3):032605, 2017.

[13] D. Bohn, R. Emunds, V. Gorzelitz, and U. Krüger. Experimental and theoretical investigations of heat transfer in closed gas-filled rotating annuli II. Journal of Turbomachinery, 118(1):11-19, 1996.

[14] D. Bohn and J. Gier. The effect of turbulence on the heat transfer in closed gas-filled rotating annuli for different Rayleigh numbers. In Proceedings of ASME 1998 International Gas Turbine and Aeroengine Congress and Exhibition, 1998. Paper No. 98-GT-542. 
[15] X. Gan, I. Mirzaee, J. M. Owen, D. A. S. Rees, and M. Wilson. Flow in a rotating cavity with a peripheral inlet and outlet of cooling air. In Proceedings of ASME 1996 International Gas Turbine and Aeroengine Congress and Exhibition, 1996. Paper No. 96-GT-309.

[16] M. P. King, M. Wilson, and J. M. Owen. Rayleigh-Bénard convection in open and closed rotating cavities. Journal of Engineering for Gas Turbines and Power, 129(2):305-311, 2005 .

[17] K. G. T. Hollands, G. D. Raithby, and L. Konicek. Correlation equations for free convection heat transfer in horizontal layers of air and water. International Journal of Heat and Mass Transfer, 18(7):879-884, 1975.

[18] J. J. Niemela, L. Skrbek, K. R. Sreenivasan, and R. J. Donnelly. Turbulent convection at very high Rayleigh numbers. Nature, 404(6780):837-840, 2000.

[19] S. Grossmann and D. Lohse. Scaling in thermal convection: a unifying theory. Journal of Fluid Mechanics, 407:27-56, 2000.

[20] D. Saini, D. Chung, and R. D. Sandberg. Direct numerical simulations of centrifugal buoyancy induced flow in a closed rotating cavity. In Proceedings of 21st Australasian Fluid Mechanics Conference, Adelaide, Australia, 2018.

[21] A. S. R. Kilfoil and J. W. Chew. Modelling of buoyancy-affected flow in co-rotating disc cavities. In Proceedings of ASME Turbo Expo 2009: Power for Land, Sea, and Air, 2009. Paper No. GT2009-59214.

[22] D. Amirante and N. J. Hills. Large-eddy simulations of wall bounded turbulent flows using unstructured linear reconstruction techniques. Journal of Turbomachinery, 137(5):051006, 2015.

[23] H. M. Blackburn and S. J. Sherwin. Formulation of a Galerkin spectral element-Fourier method for three-dimensional incompressible flows in cylindrical geometries. Journal of Computational Physics, 197(2):759-778, 2004.

[24] G. E. Karniadakis, M. Israeli, and S. A. Orszag. High-order splitting methods for the incompressible Navier-Stokes equations. Journal of Computational Physics, 97(2):414443, 1991.

[25] J. Boussinesq. Théorie de l'écoulement tourbillonnant et tumultueux des liquides dans les lits rectilignes à grande section, volume 1. Gauthier-Villars, 1897.

[26] F. Gao, W. Ma, G. Zambonini, J. Boudet, X. Ottavy, L. Lu, and L. Shao. Large-eddy simulation of 3-D corner separation in a linear compressor cascade. Physics of Fluids (1994-present), 27(8):085105, 2015.

[27] F. Gao, J. W. Chew, and D. B. Pitz. Numerical study of buoyancy-driven flow in a closed rotating annulus. In Proceedings of Global Power and Propulsion Society, 2019. Paper No. GPPS-BJ-2019-0034.

[28] R. M. Kerr. Energy budget in Rayleigh-Bénard convection. Physical Review Letters, 87(24):244502, 2001. 
[29] R. P. J. Kunnen, B. J. Geurts, and H. J. H. Clercx. Turbulence statistics and energy budget in rotating Rayleigh-Bénard convection. European Journal of Mechanics - B/Fluids, 28(4):578-589, 2009.

[30] D. B. Pitz. Direct and large-eddy simulation of buoyancy-induced flows in rotating cavities. $\mathrm{PhD}$ thesis, University of Surrey, 2018.

[31] S. Saha, C. Chin, H. M. Blackburn, and A. S. H. Ooi. The influence of pipe length on thermal statistics computed from DNS of turbulent heat transfer. International Journal of Heat and Fluid Flow, 32(6):1083-1097, 2011.

[32] E. A. Spiegel and G. Veronis. On the Boussinesq approximation for a compressible fluid. The Astrophysical Journal, 131:442, 1960. 